\title{
Evolutionary competition between boundedly rational behavioral rules in oligopoly games
}

\author{
Lorenzo Cerboni Baiardi ${ }^{\mathrm{a}, *}$, Fabio Lamantia ${ }^{\mathrm{b}}$, Davide Radi $^{\mathrm{c}}$ \\ ${ }^{a}$ DiSBeF, University of Urbino Carlo Bo, Urbino (Italy) \\ ${ }^{b}$ DESF, University of Calabria, Rende (Italy) and School of Social Sciences, University of Manchester (UK) \\ ${ }^{c}$ Department of Management, Marche Polytechnic University, Ancona (Italy)
}

\begin{abstract}
In this paper, we propose an evolutionary model of oligopoly competition where agents can select between different behavioral rules to make decisions on productions. We formalize the model as a general class of evolutionary oligopoly games and then we consider an example with two specific rules, namely Local Monopolistic Approximation and Gradient dynamics. We provide several results on the global dynamic properties of the model, showing that in some cases the attractor of the system may belong to an invariant plane where only one behavioral rule is adopted (monomorphic state). The attractors on the invariant planes can be either strong attractors or weak attractors. However, we also explain why the system can be in a state of Evolutionary Stable Heterogeneity, where it is more profitable for the agents to employ both heuristics in the long term (polymorphic state).
\end{abstract}

JEL classification: L13, D83, C61, C73

Keywords: Oligopoly, Local Monopolistic Approximation, Gradient dynamics, bounded rationality, evolutionary games.

\section{Introduction}

It is well known that the solution concept adopted in oligopoly theory is the Cournot-Nash equilibrium. In particular, the Cournot equilibrium, proposed by Cournot in 1838, is the first example of Nash equilibrium, which is a concept elaborated by Nash in 1950 for general $N$-person games. When all agents are in such an equilibrium, no unilateral deviation by a single agent is profitable and therefore no one has an incentive to deviate from that state. However, the possibility to reach the Nash equilibrium is not an obvious achievement. In real-world situations, firms are complex organizations that elaborate particular 'behavioral rules' to make their decisions regarding quantities to produce, prices, $R \& D$ activities, business strategies, advertisement, etc. If 'better' rules are currently available, it seems reasonable that the firm's management would update the previous adopted rules in order to improve the overall 'performance' of the firm.

In the economic literature, several studies have been proposed to fill the gap between the theory of oligopoly and a more realistic description of competition. As for this aspect, [1] presents an interesting example with a Cournot duopoly where ex-ante identical firms can employ different behavioral rules to set the quantities to produce. The economic structure of the underlying game is particularly simple, with homogeneous goods, linear demand and quadratic production costs. The fitness of each behavioral rule at each time period is assessed by considering average payoffs obtained by pairs of firms that are randomly matched to play the game. An evolutionary mechanism based on average profits regulates the distribution of the various rules over time. [1] concentrates on the comparison between a cheap 'Best Reply' rule and a costly 'Nash' rule and shows that endogenous fluctuations and complicated dynamics may arise, mainly due to the dominance of Best Reply behavior in a neighborhood of the Nash equilibrium because of information

\footnotetext{
* Corresponding author

Email addresses: 1.cerbonibaiardi@campus.uniurb.it (Lorenzo Cerboni Baiardi), lamantia@unical.it (Fabio Lamantia), d.radi@univpm.it (Davide Radi)
} 
costs. Also 2 presents a similar setup with linear demand and linear production costs but with random matching of $N$ firms at a time. On the basis of past performance, these firms decide to switch between costly rational and cheap boundedly-rational expectation rules on aggregate output of their rivals. In this framework, 2] finds that the classic Theocharis' result [3] on the instability of the Nash equilibrium with more than three firms is also confirmed qualitatively in an evolutionary setting.

In this paper, we firstly propose a general framework for dealing with evolutionary oligopoly models with different behavioral rules and show some general properties of such models. Afterwards, we examine a particular example with two behavioral rules proposed in the oligopoly literature to model agents with boundedly rational expectations in forecasting next period outputs. One rule is the so-called Local Monopolistic Approximation (LMA), which was first proposed in [4 to model a dynamic oligopoly where demand is not known by firms but estimated at each time period through market experiments, see also [5. 4] shows that the dynamics generated through LMA behavior converges to a Nash equilibrium also in cases where the classic Best Reply dynamics fail to converge to it. The second rule is the Gradient $(\mathrm{G})$ dynamics, which was first proposed in [6], to model the behavior of boundedly rational duopolists who decide their production in order to improve their profits over time. [6] shows under which conditions this adjustment preserves the convergence to the Nash equilibrium of the game and the possible asymptotic dynamics of the system. The main feature of firms' behavioral rules considered in this paper is that they do not require 'global' information on the demand function, even though they need 'some' information about it. It follows that in this evolutionary game firms adopt less cognitively demanding heuristics than in the most related contributions, such as [1, 2] and 7], where at least one behavioral rule employs global knowledge of the demand function.

A Cournot oligopoly game populated by firms that adopt rules based on limited knowledge of the demand function is of interest from an economic prospective, as demand function may often be difficult to be estimated, as pointed out in early contributions on learning of the demand function, see, e.g. [8] and [9], as well as in recent contributions, see, e.g., [4] and [5]. As evidence of this, it is worthwhile pointing out that an oligopoly game with heuristics analogous to the one here considered has been proposed in [10] under Bertrand competition and nonlinear demand. In that paper, some firms use Least Square Learning, wrongly assuming that demand is linear and does not depend on competitors' prices (analogously to LMA firms), and other firms use Gradient learning (in prices). Nevertheless, in both contributions the possibility to converge to a stable equilibrium depends on the parameters configuration and the presence of heterogeneous learning mechanisms may lead to non stationary dynamics.

In the proposed evolutionary oligopoly, an information cost can be associated to each behavioral rule, similarly to [1] and 2]. Differently from these papers, however, it is important to underline that in the model here considered, rules can not be ranked according to their 'degree' of rationality or to the amount of information required. Different rules are just different ways to decide production plans over time. With this in mind, different information costs in this model can be also interpreted as a bias for selecting one particular strategy over the other, as specified more precisely below. We assume a simple economic setup, with homogeneous goods and linear production costs. The selling price is obtained through isoelastic demand, widely used in these kinds of models. Moreover, an endogenous switching of behavioral rules (replicator equation) takes place according to the performance of each rule ${ }^{1}$

With respect to the dynamic properties, we detect that if the Nash equilibrium of the game is stable under any considered behavioral rule, then the asymptotic behavior of the model is very simple. In general, the cheapest behavioral rule will prevail and all agents will end up producing the Nash equilibrium quantity. In this case, the standard results of oligopoly theory are retrieved by the evolutionary model. However, intriguing questions - from an economic as well as mathematical point of view - arise when the Nash equilibrium is unstable at least under one behavioral rule. In these cases, the principal points of investigation are the following:

- What is the most likely short-run and asymptotic production of the industry when firms employ different behavioral rules but fail to converge to a Nash equilibrium?

\footnotetext{
${ }^{1}$ Often heterogeneity among players is exogenously assumed in the model. For instance, [1] considers an heterogeneous duopoly with a firm employing Local Monopolistic Approximation versus a firm employing Gradient dynamics with nonlinear demand. However, differently from this paper, 11] assumes that each firm can not change the adopted behavioral rule over time.
} 
- What is the long term distribution of the behavioral rules in the population of firms? Can behavioral heterogeneity arise endogenously as a result of interaction among identical firms switching between different heuristics?

- What is the effect of different information costs of behavioral rules on the asymptotic dynamics of the system (or, equivalently, of the presence of a bias in the population towards a particular behavioral rule)?

In this paper, we try to shed some lights on the previous questions. In doing so, we propose a standard method to analyze this family of evolutionary models, that includes the case of competition between LMA and Best Reply rules in [7] as a special case. With this respect, we are mainly interested in the dynamic scenarios that open up when instability of the Nash equilibrium occurs. Moreover, the particular example of evolutionary selection of behavioral rules analyzed herein ( $G$ and LMA rules) extends the stream of investigation started in [7 to the case of behavioral rules for which there is no clear hierarchy between them in terms of degree of required information. Before addressing the complete evolutionary model, we study some important benchmark cases with fixed fractions of agents employing the behavioral rules. Among the possible cases, two benchmarks here are particularly important: 1) the model with all LMA-players and 2) the analogous case with all G-players. In these cases, the dynamics on the two planes (all LMA or all G) is described by two triangular maps, for which we can obtain analytically important properties on the global dynamics, such as measuring the basin of attraction of the Nash equilibrium in these planes. With respect to these benchmarks, when all firms adopt the LMA rule, the Nash equilibrium becomes unstable when the number of firms is sufficiently high and a stable attractor different from the Nash equilibrium may exist. On the contrary, when all firms adopt the G rule the Nash equilibrium can be stable (depending on the parameters configuration) or, when it is unstable, the oligopoly becomes infeasible.

The global analysis of the dynamics reveals that the main differences between [7] and this paper are due to the differences between the G rule and the Best Reply rule. Specifically, the Best Reply rule has stability properties that are analogous to those of the LMA rule, i.e. instability of the Nash equilibrium occurs if the number of firms is sufficiently high. In the light of this, 7] shows that when the Best Reply rule is more expensive than the LMA rule, then it can become evolutionary dominant (all agents adopt it) only when the Nash equilibrium becomes unstable. On the contrary, here we show that when the G rule is more expensive than the LMA rule, it is never evolutionary dominant. Moreover, even if it is the cheapest rule, it dominates only when the Nash equilibrium is stable.

The analysis on invariant planes, where only one strategy is played by firms, is particularly important for understanding the complete evolutionary model, since these two planes are invariant for the model under replicator dynamics. Thus, an attractor on these planes could also be an attractor for the complete model with evolutionary switching of rules. When this happens, the population is in a monomorphic state, as all agents employ the same heuristic. Here it is meaningful to study whether this indeed happens once evolutionary pressure is introduced. In fact, if in the population only one behavioral rule is available, it is obvious that all agents must use that rule. However, we want to study whether the introduction of an alternative rule (a mutation) spreads over the population or not. From a mathematical point of view, this analysis can be carried out by studying under which conditions an attractor on an invariant plane is also an attractor for the evolutionary model. This consists in an analysis of the transverse stability of the attractors on invariant planes.

In addition, we show that attractors outside these invariant planes can exist and how they are generated through particular codimension-two bifurcations. These attractors are particularly important from an economic point of view, since they provide evidence that it might be profitable for agents to behave differently than similarly. So, not only can behavioral heterogeneity endogenously arise through competition, but it can also be the result of the long-run interaction between agents. We call this phenomenon Evolutionary Stable Heterogeneity, since evolutionary pressure selects the best behaviors and it turns out that the presence of both behavioral rules may guarantee, in the long run, an improvement of aggregate welfare to producers, measured by producer surplus. In other words, in some cases it might be more profitable for the firms to be heterogeneous, and employ different heuristics, than to be homogeneous. As a consequence, polymorphisms in the population emerges in the adoption of behavioral rules.

The paper is organized as follows. Section 2 introduces the problem and provides some general properties of the evolutionary oligopoly model. Section 3 illustrates the specific example considered in this paper, 
which is the competition between the Local Monopolistic Approximation rule and the Gradient rule. We first address the non-evolutionary version of the model and obtain some useful results on global dynamics that are important in the last part of the paper. Then we study the evolutionary model and provide some results on transverse stability of attractors located on invariant planes. Finally, we describe the emergence of inner attractors, where agents' behavioral heterogeneity arises endogenously through evolutionary pressure. Section 4 concludes.

\section{The general model}

Let us consider an oligopoly market with $N$ ex-ante identical firms that produce homogeneous goods. We assume that the set of strategies is a nonempty compact and convex set of $\mathbb{R}^{N}$ and each firm's profit is concave in its own strategy ${ }^{2}$ These assumptions guarantee that a Nash equilibrium exists, see [12. The inverse demand function, which specifies the selling price $p(t)$ as a function of produced quantities, is unknown by the agents. For this reason, firms conceive different behavioral rules for setting their next period productions. For the sake of the argument, let us consider that only two different behavioral rules are available and denote by $x_{i}(t)$ the production at time $t$ by a generic firm adopting rule $i=1,2 \sqrt{3}$ All firms employ the same technology and bear the same production cost $C(x)$.

Behavioral rule $i$ entails a fixed 'information' cost $K_{i} \geq 0$. At time $t$, the first behavioral rule is distributed with frequency $r(t) \in[0,1]$ among the firms and, obviously, the second rule with complementary frequency $1-r(t)$.

A behavioral rule can be defined as a rule that specifies next period production $x_{i}(t+1)$ as a function of the current quantities $450, i, j=1,2$, as well as of the frequency $r(t)$, i.e. ${ }^{5}$

$$
x_{i}(t+1)=H_{i}\left(x_{1}(t), x_{2}(t), r(t)\right) ; \quad i=1,2
$$

In the following, we consider behavioral rules that are stationary at any symmetric Nash equilibrium of the underlying game. More precisely, we assume that if the industry is at a Nash equilibrium, each behavioral rule prescribes to stay at that equilibrium, regardless of the distribution $r(t)$. By assumption, Nash equilibria of the game always exist. In particular, since agents are homogeneous, a symmetric Nash equilibrium exists, which is characterized by the same production by all agents, i.e. $x_{1}^{*}=x_{2}^{*}=x^{*} 6$ The stationary property at a symmetric equilibrium can be thus expressed as

$$
x^{*}=H_{i}\left(x^{*}, x^{*}, r\right) ; \quad i=1,2
$$

Stated differently, a symmetric Nash equilibrium is a fixed point of the two-dimensional map (1) for any $r \in[0,1]$.

Now consider the profit obtained by employing behavioral rule $i$ :

$$
\pi_{i}(t)=[p(t)-C(t)] x_{i}(t)-K_{i}
$$

and assume that firms can observe this quantity and switch, from period to period, to the more profitable behavioral rule, thus modifying the next-period distribution of behavioral rules. In the following, we adopt

\footnotetext{
${ }^{2}$ In particular, the case with isoelastic demand and linear costs, which is developed in the next Section, satisfies these assumptions.

${ }^{3}$ In the general setup, it is easy to generalize to $M$ different behavioral rules. However, we present the idea with only two different rules, as in the second part of this paper such an example is developed. Another example with two rules is proposed in [7].

${ }^{4}$ In general, a behavioral rule can also incorporate older information through a 'memory' term, see [7 for details. However, we do not consider this issue in this paper.

${ }^{5}$ Notice that in principle a behavioral rule $H_{i}$ should have $N+1$ arguments, i.e. the quantities by the $N$ oligopolists and the fraction $r$. However, if agents that employ the same behavioral rule set the same quantities then each behavioral rule only depends on quantities of representative agents of the different groups. This reflects in the notation, with a slight abuse of it.

${ }^{6}$ This class of behavioral rules includes as a particular case the so called unbiased rules, see, e.g., 1], which are characterized for having the symmetric Nash equilibrium $x^{*}$ as their unique equilibrium quantity.
} 
the exponential replicator model, which was firstly proposed by [13. (see also [14] and [15] and [16] for an application in oligopoly theory), which assumes the form

$$
r(t+1)=\frac{r(t) e^{\beta \pi_{1}(t)}}{r(t) e^{\beta \pi_{1}(t)}+[1-r(t)] e^{\beta \pi_{2}(t)}}=\frac{r(t)}{r(t)+[1-r(t)] e^{\beta\left[\pi_{2}(t)-\pi_{1}(t)\right]}}
$$

In (4) $\beta \geq 0$ is the intensity of choice, which measures how sensitive the players are at selecting profitincreasing behavioral rules. The minimum value $\beta=0$ corresponds to the case with fixed fractions, being $r(t+1)=r(t)=r$. The other extreme case, $\beta=\infty$, corresponds to a situation where all firms immediately switch to the behavioral rule showing a (even negligible) better performance, i.e. $r(t) \rightarrow 1$ if $\pi_{1}(t)>\pi_{2}(t)$ and $r(t) \rightarrow 0$ if $\pi_{1}(t)<\pi_{2}(t)$.

Coupling the behavioral rules in (1), which specify quantity dynamics, with the evolutionary dynamics in (4), a three-dimensional map $T$ is defined in the phase space $(x, y, r) \in A \subseteq \mathbb{R}_{+}^{2} \times[0,1]$, where $\mathbb{R}_{+}=[0,+\infty)$.

The choice of a specific evolutionary model, namely the exponential replicator, originates from its interesting properties. First of all, the strictly monotone transformation $\pi_{i}(t) \rightarrow e^{\pi_{i}(t)}$ guarantees that the fractions obtained through (4) are always contained in the interval $[0,1]$ even when $\pi_{i}(t)<0$. Another important property of (4) concerns the role of invariant planes $r=0$ and $r=1$, where only one pure strategy is employed ( $H_{2}$ or $H_{1}$ respectively). On these planes, the dynamics are governed by the two-dimensional restrictions of $T$ on them. From an economic point of view, this fact has the obvious interpretation that absent behaviors remain absent. However, the introduction of a mutation in agents' behavior may spread over the population or may be reabsorbed. This phenomenon can be ascertained through the study of transverse stability of the attractors on the invariant planes. In general, an attractor on one of these two-dimensional restrictions of the phase space may be transversely stable, so that it attracts trajectories starting outside the restriction, i.e. from $r(0) \in(0,1)$; in this case, the attractor on the restriction is also an attractor of the three-dimensional map $T$. Otherwise, the attractor on the restriction is transversely unstable, so that it might not be reached by trajectories coming from inside the phase space, but only with initial conditions $r(0)=0$ or $r(0)=1$.

From (2), it follows that if productions are at a symmetric Nash equilibrium level $x^{*}$ and $r=0, r=1$ or $r^{*} \in(0,1)$ such that $\pi_{1}=\pi_{2}$, then the points $E_{0}=\left(x^{*}, x^{*}, 0\right), E_{1}=\left(x^{*}, x^{*}, 1\right)$ or $E=\left(x^{*}, x^{*}, r^{*}\right)$ are equilibria for the three-dimensional map $T$. In addition, when the Nash equilibrium is stable with respect to the quantity adjustments (1) only, then it is easy to characterize the asymptotic behavior of the evolutionary map $T$. In fact, regardless of the specific behavioral rules considered, the following stability properties hold:

Proposition 1. Consider the dynamical system $T$ defined by (1) and (4) with $\beta>0$ and assume that the symmetric Nash equilibrium of quantity dynamics under constant $r$ is locally asymptotically stable $\forall r \in[0,1]$, $\left(\forall r \in[0,1]\left(x^{*}, x^{*}\right)\right.$ is a locally asymptotically stable fixed point of the two-dimensional map (1) with $\left.r(t)=r\right)$. The followings hold:

- If $K_{1}=K_{2}$, then a continuum of equilibrium points $E_{*}$ exists along the segment $E=\left(x^{*}, x^{*}, r\right)$, with $r \in[0,1]$. Each fixed point filling this invariant segment is stable.

- If $K_{1} \neq K_{2}$, then the segment $E=\left\{\left(x^{*}, x^{*}, r\right) \in \mathbb{R}_{+}^{2} \times[0,1]\right\}$, is invariant for $T$ and only the two extreme points of the segment $E$ are equilibria, namely

$$
E_{0}=\left(x^{*}, x^{*}, 0\right) \quad \text { and } \quad E_{1}=\left(x^{*}, x^{*}, 1\right)
$$

in which all agents adopt the same behavioral rule, which is $H_{2}$ or $H_{1}$ respectively. When $K_{1}<K_{2}$ $\left[K_{1}>K_{2}\right]$ equilibrium $E_{1}\left[E_{0}\right]$ is locally asymptotically stable, whereas $E_{0}\left[E_{1}\right]$ is unstable.

Proof. Assume that firms of either type produce the Nash equilibrium quantity $x^{*}$. Then, for any $r \in[0,1]$, the difference in their profits is given by the information costs, $\pi_{1}-\pi_{2}=K_{2}-K_{1}$ (see (3)). Therefore, if fixed information costs are equal, i.e. $K_{1}=K_{2}$, the replicator equation in 4 reduces to $r(t+1)=r(t)$ so that any point of the form $E=\left(x^{*}, x^{*}, r\right), r \in[0,1]$, is a fixed point for map $T$.

Instead, if $K_{1} \neq K_{2}$, then at any point of $E$, it is $\pi_{2} \neq \pi_{1}$, so that the stationary condition can be satisfied only at the boundary points $E_{0}=\left(x^{*}, x^{*}, 0\right)$, and $E_{1}=\left(x^{*}, x^{*}, 1\right)$, with all agents employing the same behavioral rule. 
Stability analysis of equilibria can be studied through the Jacobian matrix, which assumes the following form

$$
J\left(x^{*}, y^{*}, r\right)=\left(\begin{array}{ccc}
\frac{\partial H_{1}\left(x^{*}, y^{*}, r\right)}{\partial x_{1}} & \frac{\partial H_{1}\left(x^{*}, y^{*}, r\right)}{\partial x_{2}} & 0 \\
\frac{\partial H_{2}\left(x^{*}, y^{*}, r\right)}{\partial x_{1}} & \frac{\partial H_{2}\left(x^{*}, y^{*}, r\right)}{\partial x_{2}} & 0 \\
\frac{\partial r(t+1)}{\partial x_{1}} & \frac{\partial r(t+1)}{\partial x_{2}} & \frac{e^{\beta\left(\pi_{1}+\pi_{2}\right)}}{\left((r-1) e^{\beta \pi_{2}}-r e^{\beta \pi_{1}}\right)^{2}}
\end{array}\right)
$$

From (2), the entries $J_{13}$ and $J_{23}$ of the Jacobian matrix are equal to zero, and the characteristic equation becomes $P(z)=\left(\frac{e^{\beta\left(\pi_{1}+\pi_{2}\right)}}{\left((r-1) e^{\beta \pi_{2}}-r e^{\beta \pi_{1}}\right)^{2}}-z\right) P_{2}(z)$, where $P_{2}(z)$ is the characteristic equation of the twodimensional model (1), whose roots are in modulus less than one by the assumption of stability of the Nash equilibrium with respect to quantity dynamics. Thus, when $\pi_{1}=\pi_{2}$, which occurs when $K_{1}=K_{2}, z_{3}=1$ is an eigenvalue of $J\left(x^{*}, y^{*}, r\right)$, and any point of the form $E=\left(x^{*}, x^{*}, r\right)$ is a stable equilibrium.

If $K_{1}<K_{2}$, it is $T(E) \subset E$ by the assumption that (1) is stationary at a symmetric Nash equilibrium. Thus, $E$ is invariant for $T$. At $r=0$, the third eigenvalue is $z_{3}=\frac{e^{\beta\left(\pi_{1}+\pi_{2}\right)}}{\left((r-1) e^{\beta \pi_{2}}-r e^{\beta \pi_{1}}\right)^{2}}=e^{\beta\left(K_{2}-K_{1}\right)} \in(1,+\infty)$, whereas at $r=1$ the third eigenvalue is $z_{3}=e^{\beta\left(K_{1}-K_{2}\right)} \in(0,1)$, thus proving the statement. The eigenvector associated to the third eigenvalue is clearly $(0,0,1)$. The case $K_{2}<K_{1}$ is analogous and left to the reader. QED

The conditions under which the symmetric Nash equilibrium is stable can be violated and some attractors can be created through bifurcations, as stated in the following corollary:

Corollary 2. Consider the dynamical system $T$ defined by (1) and (4) with $\beta>0$. When the conditions for local asymptotic stability of the Nash equilibrium $\left(x^{*}, x^{*}\right)$ are broken in (1) with $r(t)=r^{*}$, the following cases occur:

- If $K_{1}=K_{2}$, an attractor can appear in $\mathbb{R}_{+} \times \mathbb{R}_{+} \times[0,1]$ (or in $\mathbb{R}_{+} \times \mathbb{R}_{+} \times(0,1)$ if $r^{*} \neq 0,1$ ) through a bifurcation of codimension 1, 2 or 3.

- If $K_{1} \neq K_{2}, r^{*}=1$ and $K_{1}<K_{2}\left[r^{*}=0\right.$ and $\left.K_{1}>K_{2}\right]$, then equilibrium $E_{1}\left[E_{0}\right]$ undergoes a bifurcation and an attractor appears in the invariant subspace $r=1[r=0]$, whereas $E_{0}\left[E_{1}\right]$ remains unstable.

Proof. The first part of the corollary follows observing that two of the roots of the characteristic polynomial associated to the Jacobian matrix of dynamical system $T$ (defined by (1) and (4) with $\beta>0$ ) computed at the fixed point $E=\left(x^{*}, x^{*}, r^{*}\right)$, coincide with the two roots of the characteristic polynomial of the Jacobian matrix of system (1) computed at the fixed point $\left(x^{*}, x^{*}\right)$ with $r(t)=r^{*}$.

The second part of the corollary follows by noting that the dynamics on the invariant plane $r=1$ (or $r=0$ ) of the dynamical system $T$ is equal to the one of system (1) with $r(t)=1$ (or $r(t)=0)$. QED

On the other hand, if the Nash equilibrium is unstable for the quantity adjustments (1) and more complex attractors exist, then the asymptotic behavior of the model becomes more complicated but also more interesting both from a mathematical as well as economic point of view. In the rest of the paper, we develop this model with two specific behavioral rules, namely the Local Monopolistic Approximation (LMA) and the Gradient $(\mathrm{G})$ rules and we investigate the main dynamic properties of the system.

\section{An example with specific behavioral rules}

In this Section, we develop the model described above in its general form. We consider as specific behavioral rules the Local Monopolistic Approximations (LMA) and the Gradient dynamics (G), proposed, respectively, in $[4$ and 6 . form

Let us consider an oligopoly with $N \geq 2$ firms that produce homogeneous goods with linear cost of the

$$
C_{i}=c \cdot q_{i}+K_{i}
$$


where $q_{i}$ is the quantity produced by firm $i, i=1, \ldots, N, c>0$ denotes the marginal cost (equal for all firms) and $K_{i} \geq 0$ represents an information cost. This latter term depends on firm's $i$ behavioral rule for setting next-period production, as specified below. Market price is a function of the overall quantity in the market. We assume that this price is determined through an isoelastic demand function, with constant elasticity equal to one:

$$
p=f(Q)=\frac{1}{Q}
$$

where $Q=\sum_{i=1}^{N} q_{i}$ is the total industry output 7 In the following we focus on dynamics such that $Q>0$. The case $Q=0$ is defined as infeasibility of the oligopoly $8^{8}$

Firms can adopt different behavioral rules to decide their production quantities, which occur at discrete time periods $t \in \mathbb{N}$. In particular, we consider the interaction between Gradient (G-)players and Local Monopolistic Approximation (LMA-)players.

At a given time $t$, the fraction of G-players is denoted by $r(t) \in[0,1]$, with the complementary fraction $1-r(t)$ employing the LMA rule 9 In the following, the output at time $t$ of a representative $\mathrm{G}$ and LMA-firm are denoted, respectively, by $x(t)$ and $y(t)$.

At time $t$, the $i$-th representative G-firm sets production in the direction that maximizes its expected profits for time $t+1$. To be more specific, consider the current profits of a representative G-firm:

$$
\pi_{G}\left(x(t), Q_{-1}(t)\right)=\left[\frac{1}{x(t)+Q_{-1}(t)}-c\right] x(t)-K_{G}
$$

where $Q_{-1}(t)$ is the current production for the rest of the industry. The term $K_{G} \geq 0$ in (7) represents a fixed 'information' cost associated to $G$ behavior. The behavioral rule adopted by a G-firm consists on setting its next period quantity as follows

$$
\begin{aligned}
x(t+1) & =\max \left[0, x(t)+\lambda x(t) \frac{\partial \pi_{G}}{\partial x(t)}\right]= \\
& =\max \left[0, x(t)+\lambda x(t)\left(\frac{Q_{-1}(t)}{\left(x(t)+Q_{-1}(t)\right)^{2}}-c\right)\right]
\end{aligned}
$$

where $\lambda \geq 0$ is the speed of adjustment (equal for all G-players).

By defining $Q_{-1}(t)$ as ${ }^{10}$

$$
Q_{-1}(t)=(N-1)\{r(t) x(t)+[1-r(t)] y(t)\}
$$

\footnotetext{
${ }^{7}$ This particular demand function is widely employed in the literature, see, e.g., [17, [18, [19, 20], 21] and [22]. In particular, isoelastic demand is obtained when a representative consumer maximizes a log-linear (or Cobb-Douglas) utility function, see 21] for details.

${ }^{8}$ Note that for $Q=0$ the demand function is undefined and therefore the oligopoly is infeasible. An alternative would be to impose that the demand function is equal to a finite amount for $Q=0$. In this case, $Q=0$ represents a stable fixed point for the game and the set of trajectories that are now infeasible would converge to such a fixed point. Nevertheless, the economic interpretation of the results would not change as in either cases $Q=0$ represents the infeasibility (or unprofitability) of the oligopoly.

${ }^{9}$ It is worth pointing out that the adoption of replicator dynamics, in whatever form, implies a random matching, see, e.g., 23. Then, $r(t)$ must be interpreted as the probability to meet a $G$-player, or, better, the frequency of $G$-players. Nevertheless, with an abuse of language, we call $r(t)$ the fraction of $G$-players.

${ }^{10}$ According to the setup here adopted, modeling both G and LMA heuristics requires to know the realized level of production. Indeed, G-firms observe their profits, which are here expressed as a function of the total level of production of the industry. At the same time, LMA-firms observe the current price, which is again expressed as a function of the realized level of production of the industry. However, this modeling setup does not provide the exact level of production of the industry but only its expected value. Thus, for modeling purposes we considered the last one as a proxy for the first one. See also [1] and [7] for the same assumption. This modeling choice implies that G-firms estimate the derivative of their own profits computed at the expected level of production (instead of estimating the derivative of their own realized profits) and LMA-firms approximate the inverse demand function in a neighbourhood of the expected level of production (instead of approximating the inverse demand function in a neighbourhood of the realized production). However, the expected level of production should be an acceptable proxy for the realized one, at least for a sufficiently high number of players. It is also worth pointing out that in the classical approach of decision theory (see [2] for a similar setup) firms select the Best Reply dynamics maximizing expected profits instead of profits of expected productions. That approach, however, would require to assume (contrary to what we do here) that firms have global knowledge of the demand function. See also 24] for a brief discussion about the issue of discreteness of the space of firms in oligopoly models.
} 
we can rewrite 8 as follows

$$
x(t+1)=\max \left[0, x(t)+\lambda x(t)\left(\frac{(N-1)[r(t) x(t)+(1-r(t)) y(t)]}{(x(t)((N-1) r+1)+y(t)((N-1)(1-r)))^{2}}-c\right)\right]
$$

Now we briefly describe the LMA rule. A representative LMA-agent sets $y(t+1)$, its production for time $t+1$, in order to maximize its expected profits at time $t+1$

$$
\max _{y(t+1) \geq 0}\left[\widetilde{p}^{e}(t+1)-c\right] y(t+1)-K_{L}
$$

where $K_{L} \geq 0$ is a fixed information cost for LMA-players.

The expected price $\widetilde{p}^{e}(t+1)$ in $(12)$ is assessed through the following calculation (see 4 for details)

$$
\widetilde{p}^{e}(t+1)=p(t)+f^{\prime}\left(Q_{-1}^{e}(t+1)\right)[y(t+1)-y(t)]
$$

where $p(t)$ and $f(Q)$ are given in (6). The expected quantity $Q_{-1}^{e}(t+1)$ is approximated by current production

$$
Q_{-1}^{e}(t+1)=Q_{-1}(t)
$$

which is given in 10 . In other words, LMA-players adopt a linear estimation for future price, where the slope of demand is correctly assessed and the increments in quantities disregard the production by the other competitors (from which the name Local Monopolistic Approximation). This is equivalent to say that an LMA-firm assumes that all other firms will produce at time $t+1$ the same quantity produced at time $t$ (as assumed in the well-known Best Reply adjustment, see [18]).

Through rule (8) or maximizing (12), a firm adopts, respectively, a G or a LMA behavior to determine its production plan for time $t+1$. By symmetry, at each time $t$, all agents with the same behavioral rule $(\mathrm{G}$ or LMA) produce the same quantity.

Notice that LMA and G-agents do not know the form of the demand function (6) nor they need to form expectations on rivals' future outputs when deciding their own production. However, they employ different informational and behavioral assumptions to determine their future productions. The generic LMA-firm: it

- is able to locally estimate $\frac{\partial f(Q)}{\partial y}$, the partial derivative of $(6)$ with respect to its own production at the present industry output level;

- is able to solve a quadratic optimization problem, which involves solving a linear equation when costs are linear (as in this paper) or quadratic.

With respect to the generic G-firm: it

- is able to locally estimate $\frac{\partial \pi_{G}}{\partial x}$, the partial derivative of its profits $(7)$ with respect to its own production at the present industry output level;

- does not solve any optimization problem, but sets quantities in the direction (increment or reduction) such that its expected profit increases.

All in all, by coupling the dynamics of quantities by $\mathrm{G}$ and LMA-agents with an exponential replicator for the evolution of the fraction of G-players, the following three dimensional map $T: A \longrightarrow A$ is obtained, where $A=\left(\mathbb{R}_{+}^{2} \backslash\left(\cup_{n=0}^{+\infty} T^{-n}(0,0, r) \cup_{n=0}^{+\infty} T^{-n}(0, y, 1) \cup_{n=0}^{+\infty} T^{n}(x, 0,0)\right)\right) \times[0,1] 11$

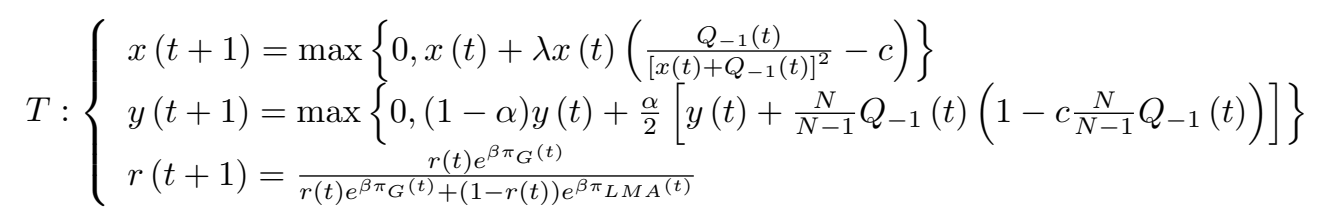

\footnotetext{
${ }^{11}$ Observe that map $T$ is undefined in the points $(x, y, r)$ that are mapped in $n$ iterations in the focal point $(0,0, r)$ because of the first component of $T$. The same holds also for the other focal point (of second type) of $T$ given by $(0, y, 1)$. The set $\cup_{n=0}^{+\infty} T^{-n}(x, 0,0)$ is not included as $(x, 0,0)$ is a point of infeasibility of the oligopoly. See [25] for details.
} 
where $Q_{-1}(t)$ is given in 10$\}$ and $\pi_{i}(t), i \in\{G, L M A\}$, are, respectively:

$$
\begin{aligned}
& \pi_{G}(t)=p(t) x(t)-\left(c x(t)+K_{G}\right)=\left(\frac{N-1}{N Q-1(t)}-c\right) x(t)-K_{G} \\
& \pi_{L M A}(t)=p(t) y(t)-\left(c y(t)+K_{L}\right)=\left(\frac{N-1}{N Q_{-1}(t)}-c\right) y(t)-K_{L}
\end{aligned}
$$

The max operator in 15 imposes nonnegativity of productions whenever a behavioral rule returns a negative quantity, so that the firm simply decides not to produce for the next time period. Differently, region $\mathbb{R}_{+}^{2} \backslash A$, where map $T$ is not defined, refers to cases of infeasibility of the oligopoly, which occurs, as previously recalled, since the demand function (6) is not defined for $Q(t)=0$. The parameters $\alpha$ and $\lambda$ in 15) represent speeds of adjustment for LMA and G-players (see [18] for details).

Summing up, model 15 depends on the following parameters:

- $N \in[2,+\infty) \cap \mathbb{N}$ (number of agents);

- $\alpha \in(0,1]$ and $\lambda \in(0,+\infty)$ (speed of adjustment of LMA and G-agents respectively);

- $c \in[0,+\infty)$ (marginal production costs);

- $K_{G} \in[0,+\infty)$ (information cost for G-agents);

- $K_{L} \in[0,+\infty)$ (information cost for LMA-agents);

- $\beta \in[0,+\infty)$ (intensity of choice in the evolutionary process).

Before addressing the complete model (15), we study a simplified version of it where evolutionary pressure is disregarded. The obtained results are then useful for understanding 15.

\subsection{A useful benchmark case}

Here we study 15 in the case $\beta=0$. This is equivalent to assume that the fraction of G-players (and consequently of LMA-players) is exogenously fixed, i.e. $r(t+1)=r(t)=r$. This benchmark case provides interesting insights on the complete model (15). With $\beta=0,15$ reduces to a two dimensional map $T_{2}: A_{2} \longrightarrow A_{2}$ where $A_{2}=\mathbb{R}_{+}^{2} \backslash \cup_{n=0}^{+\infty} T_{2}^{-n}(0,0):{ }^{12}$

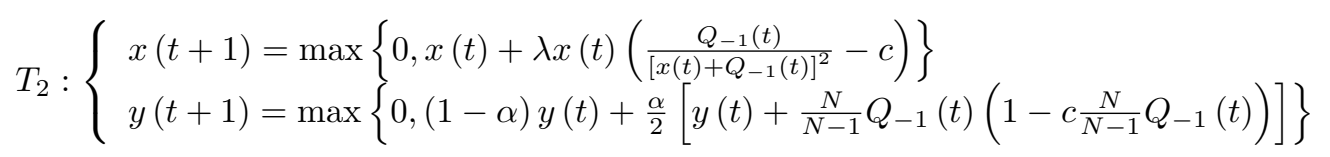

where $Q_{-1}(t)=(N-1)\{r x(t)+(1-r) y(t)\}$, see also 10 .

Observe that the game with all G or all LMA-players has the same (Cournot-)Nash equilibrium, as proved in [6] and [4]. Therefore the (unique) Nash equilibrium $E_{*}=\left(x^{*}, y^{*}\right)$ is characterized by the same quantity for both kinds of agents:

$$
x^{*}=y^{*}=\frac{N-1}{c N^{2}}
$$

In addition, $E_{*}$ is a fixed point of 17 . The following proposition sums up the stability properties of 17 (3 $^{13}$

Proposition 3. Consider the dynamical system $T_{2}$ in (17) and its non-trivial equilibrium $E_{*}$ in (18).

- If $r=0$ (all LMA-players), then $E_{*}$ is a stable node provided that $2 \leq N<1+\frac{4}{\alpha}$ and $\lambda<\frac{N}{c}$ :

1. $E_{*}$ loses stability through a period doubling bifurcation at $\lambda=\frac{N}{c}$ with $2 \leq N<1+\frac{4}{\alpha}$. The 2-cycle that appears belongs to the subspace $y=y^{*}$;

\footnotetext{
${ }^{12}$ It is worth noting that for the case $r=1$ it is $T_{2}: A_{2} \longrightarrow A_{2}$ where $A_{2}=\mathbb{R}_{+}^{2} \backslash \cup_{n=0}^{+\infty} T_{2}^{-n}(0, y)$, while for the case $r=0$ it is $T_{2}: A_{2} \longrightarrow A_{2}$ where $A_{2}=\mathbb{R}_{+}^{2} \backslash \cup_{n=0}^{+\infty} T_{2}^{-n}(x, 0)$.

${ }^{13}$ The first bullet point of Proposition 3 has already been proved in [7.
} 
2. $E_{*}$ loses stability through a period doubling bifurcation at $N=\max \left\{2,1+\frac{4}{\alpha}\right\}$ with $\lambda<\frac{N}{c}$. The 2-cycle that appears belongs to $\mathbb{R}_{+}^{2}$.

3. At $N=\max \left\{2,1+\frac{4}{\alpha}\right\}$ and $\lambda=\frac{N}{c}$, both eigenvalues of the Jacobian matrix of (17) calculated at $E_{*}$ are on the unit circle.

- if $r=1$ (all G-players), then $E_{*}$ is a stable node for all $N \geq 0$ provided that $\lambda<\frac{2}{c}$; for $\lambda>\frac{2}{c}$ the map is eventually defined only on $E_{*}$ : any other initial condition will lead the map to the focal point $(0,0)$;

- if $0<r<1$ (mixed population state) and

$$
2 \leq N<N_{b}=\frac{5-2 r}{1-r}
$$

then $E_{*}$ is a stable node provided that the following condition holds:

$$
\lambda<\lambda^{*}(\alpha)=\frac{2 N(\alpha(1-2 r-N(1-r))+4)}{c(r(\alpha+4)(N-2)-2 \alpha(N-1)+8)}
$$

- if $0<r<1$ (mixed population state) and $N>N_{b}$, then $E_{*}$ is a stable node provided that condition (20) holds with the following additional condition:

$$
\alpha<\alpha^{*}=\frac{4}{N(1-r)+2 r-1}
$$

- For $\lambda^{*}(\alpha)>0$ and $\alpha<\alpha^{*}, E_{*}$ loses stability at $\lambda=\lambda^{*}(\alpha)$ through a period-doubling bifurcation.

- For $r \neq 1$, the map $T_{2}$ has the additional equilibrium $E_{x=0}=\left(0, \frac{N-1-N r}{c N^{2}(1-r)^{2}}\right)$. If $E_{x=0}$ is asymptotically stable, then it must be that $\alpha<\frac{4}{N(1-r)-1}$.

Proof. Consider the Jacobian matrix of (17) computed at equilibrium (18), which is given by

$$
J\left(E_{*}\right)=\left(\begin{array}{cc}
\frac{N-c \lambda(2+(N-2) r)}{N} & -\frac{c(N-2)(1-r) \lambda}{N} \\
-\frac{(N-2) r \alpha}{2} & \frac{1}{2}((1-N(1-r)-2 r) \alpha+2)
\end{array}\right)
$$

For $r=0$ and $r=1$, the result follows immediately because $J\left(E_{*}\right)$ becomes a triangular matrix. For the stability of the equilibrium $E_{*}$ when $0<r<1$, it is required that the following system of inequalities (known as Schur or Jury's conditions, see e.g. [26], 27], 28]) are satisfied:

$$
\left\{\begin{array}{l}
1+\operatorname{tr} J\left(E_{*}\right)+\operatorname{det} J\left(E_{*}\right)>0 \\
1-\operatorname{tr} J\left(E_{*}\right)+\operatorname{det} J\left(E_{*}\right)>0 \\
1-\operatorname{det} J\left(E_{*}\right)>0
\end{array}\right.
$$

where $\operatorname{tr} J\left(E_{*}\right)$ and $\operatorname{det} J\left(E_{*}\right)$ are, respectively, the trace and the determinant of 22). By straightforward calculations, it is possible to verify the following statements. Condition $1-\operatorname{det} J\left(E_{*}\right)=0$ is never satisfied if the other conditions in $(23)$ hold, thus ruling out that $E_{*}$ can lose stability through a Neimark-Sacker bifurcation. If condition (21) holds, then it is $1-\operatorname{tr} J\left(E_{*}\right)+\operatorname{det} J\left(E_{*}\right)>0$. Assuming condition (21) and imposing $1-\operatorname{det} J\left(E_{*}\right)>0$, we can solve for $1+\operatorname{tr} J\left(E_{*}\right)+\operatorname{det} J\left(E_{*}\right)=0$, thus obtaining the bifurcation value $\lambda=\lambda^{*}(\alpha)$ in 20 . These conditions are necessary for a flip bifurcation of the two-dimensional map (17) to occur. The sufficient conditions can be verified by standard calculations ${ }^{14}$ Moreover, it is worth noting that $\lambda^{*}\left(\alpha^{*}\right)=0$, and that $\lambda^{*}(\alpha)>0$ for $\alpha<\alpha^{*}$ and $\lambda^{*}(\alpha)<0$ for $\alpha>\alpha^{*}$, at least in a neighborhood of $\alpha^{*}$. This confirms that equilibrium $E_{*}$ loses stability only at $\lambda=\lambda^{*}(\alpha)$. In fact, condition (21) ensures that in 20 it is $\lambda^{*}(\alpha)>0$. Observe that in (21) it is $\alpha^{*}>0$. When the number of players is such that $2 \leq N<N_{b}=\frac{5-2 r}{1-r}$, then the denominator in the right-hand side of 21$]$ is smaller than 4 , so that condition

\footnotetext{
${ }^{14}$ We provide evidence of the 2-cycle by numerical simulations showing its existence.
} 
21) is always satisfied, being $\alpha \leq 1$. In other words, if the number of players is smaller than $N_{b}$, then condition (20) alone guarantees that the Nash equilibrium $E_{*}$ is a stable node, being $\lambda^{*}(\alpha)>0$ for all $\alpha \in(0,1]$.

With respect to equilibrium $E_{x=0}$, by employing the component $x(t)+\lambda x(t)\left(\frac{Q_{-1}(t)}{\left[x(t)+Q_{-1}(t)\right]^{2}}-c\right)$ of the first equation in (17), the Jacobian matrix assumes the following triangular form

$$
J\left(E_{x=0}\right)=\left(\begin{array}{cc}
1+\frac{c(N(r-2)+1) \lambda}{(N-1)(N(r-1)+1)} & 0 \\
-\frac{(N(r-1)+2) r \alpha}{2(r-1)} & 1+\frac{1}{2}((N(r-1)+1) \alpha)
\end{array}\right)
$$

whose eigenvalues are the entries along the diagonal. It is easy to show that it is impossible for both eigenvalues to be inside the unitary circle. However, when the constraint $x=0$ is active because of the Max in 17), then the entry in the first row and first column of $J\left(E_{x=0}\right)$ becomes zero, because the map is constant. In this case, one eigenvalue is always $z_{1}=0$ and the other eigenvalue is $z_{2}=\frac{1}{2}((N(r-1)+1) \alpha+2)$. Condition $z_{2} \in(-1,1)$ reduces to $\alpha<\frac{4}{N(1-r)-1}$, which is a necessary condition for the stability of $E_{x=0}$. QED

The stability analysis of the two-dimensional model (17) in the previous proposition underlines two important differences of the considered behavioral rules with respect to the convergence to the Nash equilibrium. In fact, under the Gradient rule this convergence is always ensured by a sufficiently low speed of adjustment, regardless of the number of firms $N$. On the contrary, the LMA rule guarantees convergence to the Nash equilibrium provided that, for a given speed of adjustment $\alpha$ of LMA behavior, the number of players is sufficiently low. Stated differently, in order to guarantee convergence to the Nash equilibrium $E_{*}$ under the LMA rule, the speed of adjustment $\alpha$ must be reduced as the number of firms in the oligopoly increases. Moreover, when LMA and G-firms interact with fixed proportion $r$ of G-firms, then it is

$$
\begin{array}{r}
\lim _{r<1, N \rightarrow \infty} \alpha^{*}=0 \\
\lim _{r<1, N \rightarrow \infty} \lambda\left(\alpha^{*}\right)=0
\end{array}
$$

so that for given values of $\alpha$ and $\lambda$, if the number of players is sufficiently high, then the presence of a positive fraction of LMA-players always destabilizes the Nash equilibrium. It is worth pointing out also the following:

Remark. Proposition 3 states that for $r=0$ the Nash equilibrium $E_{*}$ is stable if both the following conditions are satisfied: $2 \leq N<1+\frac{4}{\alpha}$ and $\lambda<\frac{N}{c}$. However, from an economic point of view, the first condition alone is sufficient to ensure that all players select the Nash quantity $y^{*}=\frac{N-1}{c N^{2}}$. In fact, at $\lambda=\frac{N}{c}$ equilibrium $E_{*}$ loses stability through a flip bifurcation so that an asymptotically stable 2-cycle appears. However, as stated in Proposition 3, this 2-cycle belongs to the subspace $y=y^{*}$, i.e. it is of the type $\left\{\left(x_{1}, y^{*}\right),\left(x_{2}, y^{*}\right)\right\}$. Therefore, although the quantity produced by G-firms changes over time, all players still produce $y^{*}$, since no G-firms are present, being $r=0$.

These analytical results unveil quite reach dynamic scenarios, which are further investigated in the following by numerical analysis. In particular, we provide a detailed investigation of all the possible scenarios of the bidimensional model here considered, with the aim to provide a better understanding of the threedimensional dynamical system.

Let us start by taking the speed of adjustment $\alpha$ of LMA-players as a bifurcation parameter. In particular, we investigate how the long-run dynamics of the model change as $\alpha$ varies in $(0,1]$ under two different dynamic scenarios. In the first one, depicted in Figure 1 , we consider a situation such that for $r=1$ (with G-firms only) equilibrium $E_{*}$ is stable, so that in the long run the level of production converges to the Nash equilibrium. According to Proposition 3 this occurs when $\lambda<\frac{2}{c}$. In this case, we observe that as the fraction $r$ of G-firms increases, the values of $\alpha$ at which the Nash equilibrium $E_{*}$ loses stability increases as well. Moreover, we observe that for $r=0$ the oligopoly becomes infeasible as $\alpha$ increases, see Figure 1(a). This is due to the fact that the oligopoly competition leads firms to stop the production and the demand function (6) is not defined when total production is zero. In the second scenario, when $\lambda>\frac{2}{c}$, whose simulations are not reported for the sake of brevity, the equilibrium $E_{*}$ is unstable for $r=1$ and, the oligopoly becomes infeasible as all the 
firms eventually decide to stop their production. In addition, as $\alpha$ increases, the oligopoly becomes infeasible also in the case of a mixed population state, i.e. $r \in(0,1)$, when $\lambda>\lambda^{*}(\alpha)$.

Let us now perform a similar analysis as $\lambda$, the reactivity of G-firms, changes. In Figure 2 the limit dynamics of $x$ and $y$ are depicted as $\lambda$ varies; in these example, the stability condition $\alpha<\alpha^{*}$ is ensured, i.e. the stability of the Nash equilibrium $E_{*}$ depends only on $\lambda^{*}$, which in turns depends on $r$. It is worth noting that if the game is played by LMA-firms only, i.e. $r=0$, when $\lambda>\lambda^{*}$ the Nash equilibrium $E_{*}$ becomes unstable but the oligopoly remains feasible, see Figure 2, panel (a). On the contrary, if the game is played by G-firms only, i.e. $r=1$, the condition $\lambda<\lambda^{*}$ determines the feasibility of the oligopoly game itself, see Figure 2. panel (c). Condition (20) for the stability of the Nash equilibrium $E_{*}$ for an intermediate level of $r=0.5$ is depicted in Figure 2 panel (b).

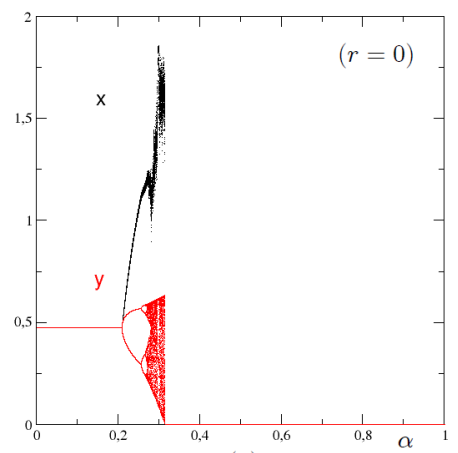

(a)

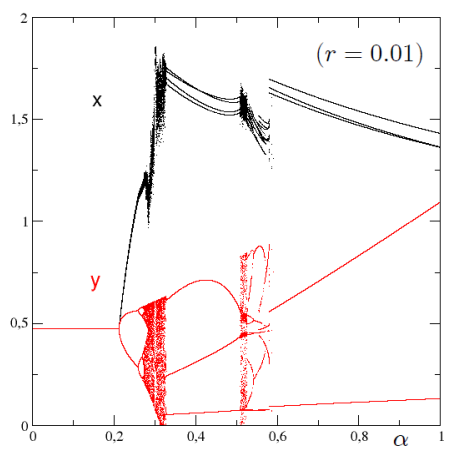

(b)

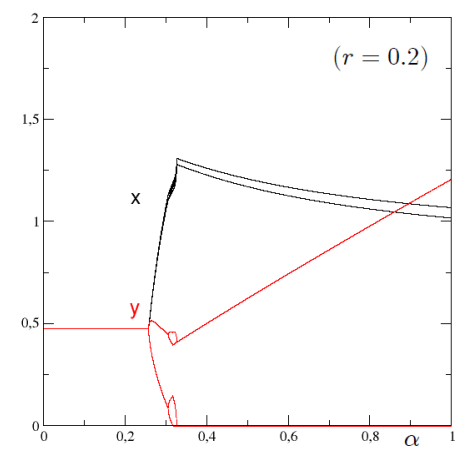

(c)

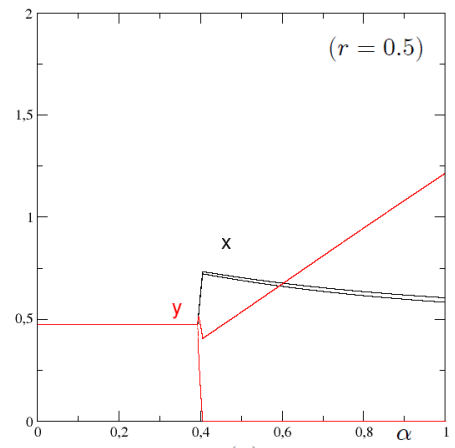

(d)

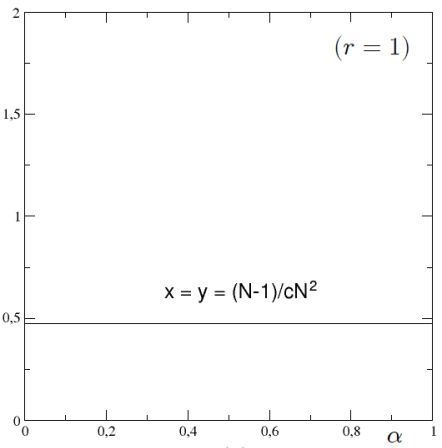

(e)

Figure 1: Bifurcation diagrams of $x$ (black) and $y$ (red) for $\alpha$ varying in $(0,1]$. Case $\lambda<\frac{2}{c}$. Panel (a) $r=0$. Panel (b) $r=0.01$. Panel (c) $r=0.2$. Panel (d) $r=0.5$. Panel (e) $r=1$. Parameters values: $\lambda=0.8, N=20, c=0.1$. Black dots in Panel (a) refer to the production level of G-firms the frequency of which is equal to zero $(r=0)$, it follows that such black dots are irrelevant for the description of the oligopoly.

Another aspect worth of investigation is the interaction between the two different behavioral rules as parameter $r \in[0,1]$ is changed. To this aim, we present several examples in Figure 3 In Panel (a), the parameters values are such that the equilibrium $E_{*}$ is stable both for $r=0$ and for $r=1$, i.e. $2 \leq N<1+\frac{4}{\alpha}$ and $\lambda<\frac{2}{c}$. We observe that in this case the stability of the Nash equilibrium occurs also for $r \in(0,1)$. In other words, when the Nash equilibrium is stable with either all LMA-firms or all G-firms, then it is stable for any $r \in(0,1)$. In Panel (b), the parameters values are such that the Nash equilibrium $E_{*}$ is unstable with all LMA-firms (i.e. $r=0)$ but it is stable with all G-firms $(r=1)$. In other words, $N>1+\frac{4}{\alpha}$ and $\lambda<\frac{2}{c}$. In this case, for small values of $r$ a complex attractor exists, while as the fraction of G-firms in the oligopoly increases, a cascade of halving bifurcations occurs and the Nash equilibrium gains stability already for relatively low values of $r$. In Panel (c), the parameters values are such that the Nash equilibrium $E_{*}$ is stable with all LMA-firms and unstable with all G-firms. In particular, parameters are such that 


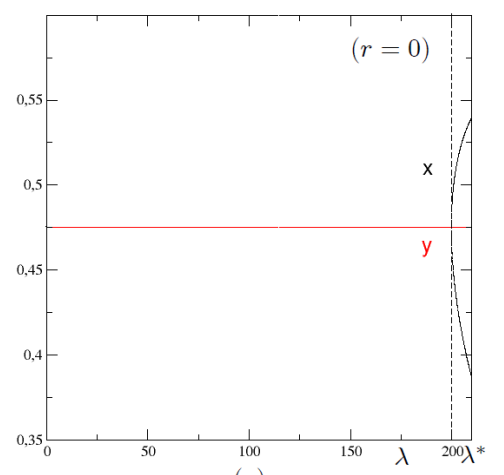

(a)

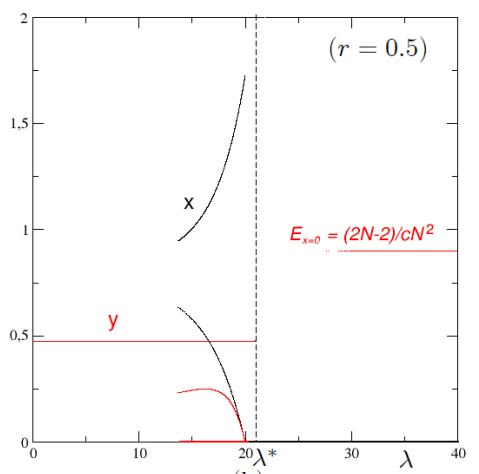

(b)

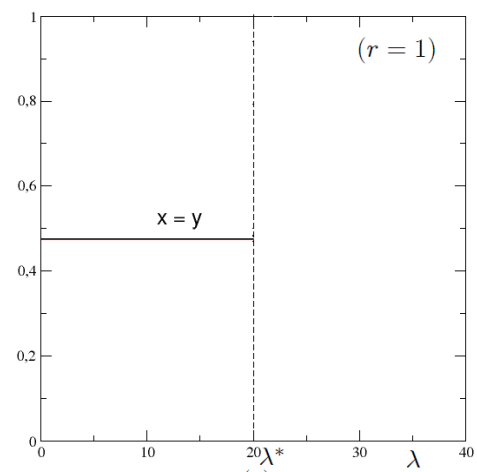

(c)

Figure 2: Bifurcation diagrams of $x$ (black) and $y$ (red) for $\lambda$ varying on (0,1]. Panel (a) $r=0$. Panel (b) $r=0.5$. Panel (c) $r=1$. Parameters values: $\alpha=0.2, N=20, c=0.1$.

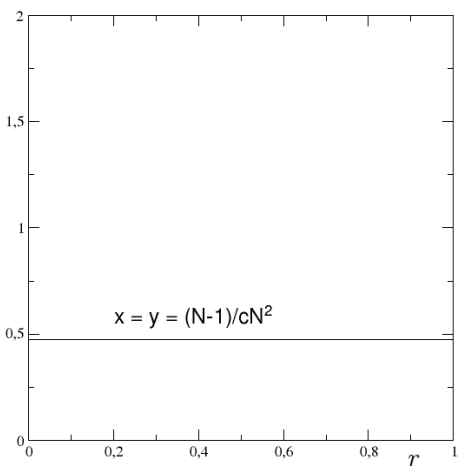

(a)

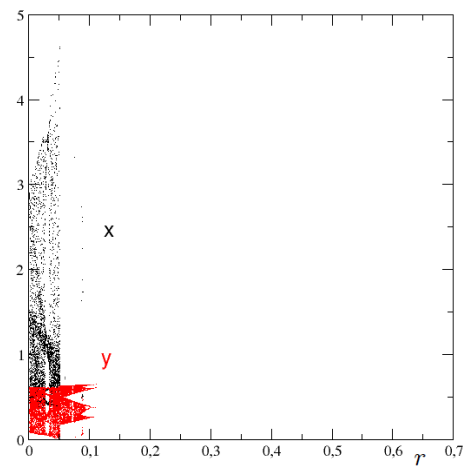

(d)

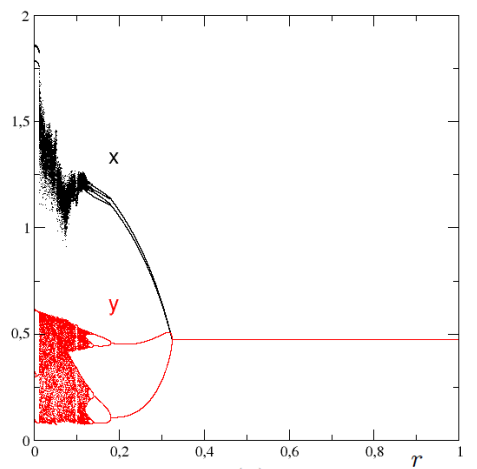

(b)

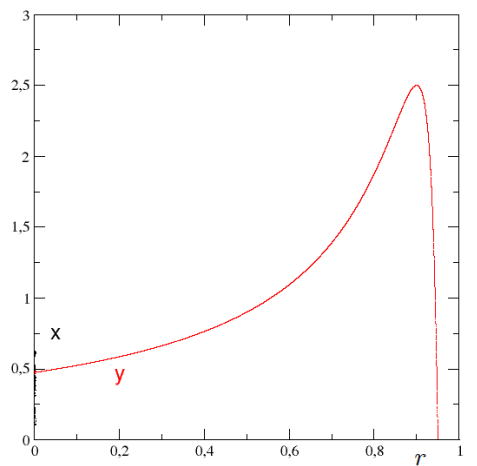

(e)

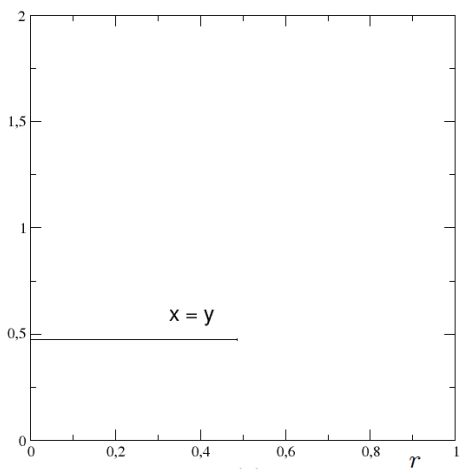

(c)

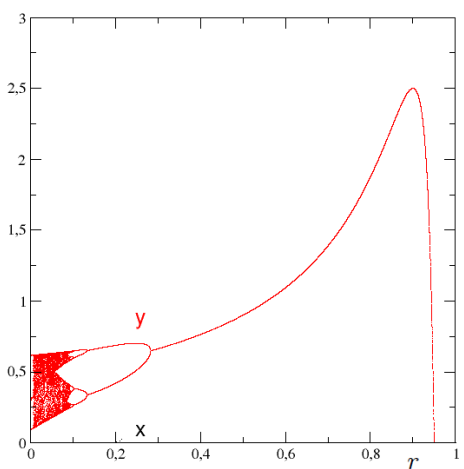

(f)

Figure 3: Bifurcation diagrams of $x$ (black) and of $y$ (red) for $r$ varying on [0,1]. Panel (a) $\alpha=0.5, \lambda=1$. Panel (b) $\alpha=0.3$, $\lambda=1$. Panel (c) $\alpha=0.2, \lambda=21$. Panel (d) $\alpha=0.3, \lambda=21$. Panel (e) $\alpha=0.2, \lambda=201$. Panel (f) $\alpha=0.3, \lambda=201$. Other parameters values: $N=20, c=0.1$.

$2 \leq N<1+\frac{4}{\alpha}$ and $\frac{N}{c}>\lambda>\frac{2}{c}$. In this case, we first observe that the Nash equilibrium is asymptotically stable when the fraction of the LMA-firms in the oligopoly is high (low $r$ ), while the oligopoly becomes infeasible, i.e. firms stop production, as the fraction of G-firms increases. In Panel (d), the parameters values are such that the Nash equilibrium is unstable with all LMA-firms as well as with all G-firms. In particular, parameters are such that $N>1+\frac{4}{\alpha}$ and $\frac{N}{c}>\lambda>\frac{2}{c}$. In this case, complex dynamics for low fraction of G-firms are observed while the oligopoly becomes infeasible as the fraction $r$ of G-firms increases. 
In Panel (e), we present the case $2 \leq N<1+\frac{4}{\alpha}$ and $\lambda>\frac{N}{c}$. It means that with all LMA-firms the Nash equilibrium is unstable, and the asymptotically stable equilibrium is the equilibrium $E_{x=0}$ defined in Proposition 3. When the fraction of G-firms is increased, this equilibrium loses stability and the oligopoly becomes infeasible. With all G-firms the oligopoly results to be infeasible as well. In Panel (f), we depicted the case $N>1+\frac{4}{\alpha}$ and $\lambda>\frac{N}{c}$, i.e. $E_{*}$ is unstable for both $r=1$ and $r=0$. Paradoxically, in this case the oligopoly game remains feasible for higher values of $r$, although two eigenvalues are outside the unit circle, if compared to the case of Panel (d), where equilibrium $E_{*}$ has only one eigenvalue outside the unit circle for $r=0$.

The investigation based on bifurcation diagrams reveals that if one starts from a situation where only one of the two heuristics is employed, either G or LMA, and this rule leads the oligopoly to converge to the Nash equilibrium $E_{*}$, then the presence of this 'stable' rule favors the convergence to the Nash equilibrium also when there is a fraction of agents adopting the other available rule.

In addition, the numerical analysis reveals another important aspect of the model, which is related to the lost of asymptotic stability of the Nash equilibrium. In particular, if the oligopoly is populated by LMA-agents only, then the production dynamics are governed by a nonlinear map, with a range of stability of the Nash equilibrium that is lower than with all G-firms; however, when this equilibrium loses stability, the oligopoly remains feasible although fluctuations of the level of production are observed. On the contrary, if the oligopoly is populated by G-firms only, then the level of production depends on a piecewise-linear map. Thus, the Nash equilibrium can be characterized by more stability if one considers the size of its basin of attraction. However, when the Nash equilibrium loses stability, then the only possibility is that of an 'infeasible' oligopoly, with all agents that stop to produce and exit the market.

\subsubsection{Global Analysis}

In this part, we provide some considerations on the global dynamics of the map $T_{2}$, which are also useful for understanding the behavior of the evolutionary model $T$ in (15). In the next proposition, we analyze the structure of the basins of attraction of the Nash equilibrium when only one behavioral rule is present, i.e. with $r=1$ (all G-firms) or $r=0$ (all LMA-firms) and compare their measure in the two cases.

Proposition 4. Consider the dynamical system $T_{2}$ in (17) and its non-trivial equilibrium $E_{*}$ in (18). Denote by $\mu(\mathcal{V})$ the measure of a region $\mathcal{V} \subseteq\left\{(x, y) \in \mathbb{R}_{+}^{2}\right\}$ and by $\mathcal{B}(V)$ the basin of attraction of an attractor $V$ of $T_{2}$. Define the following sets:

$$
\begin{aligned}
& \mathcal{B}_{1}:=\left\{(x, y) \in \mathbb{R}_{+}^{2} \mid x \geq \frac{\lambda}{c \lambda-1} \frac{N-1}{N^{2}}\right\}, \\
& \mathcal{B}_{2}:=\left\{(x, y) \in \mathbb{R}_{+}^{2} \mid y \geq \frac{2-\alpha+\alpha N}{\alpha c N^{2}}\right\}, \text { and } \\
& \mathcal{B}_{3}:=\left\{(x, y) \in \mathbb{R}_{+}^{2} \mid x^{2}+(N-1)^{2} y^{2}+(N-1) x y-\frac{\lambda(N-1)}{\lambda c-1} y \leq 0\right\}
\end{aligned}
$$

Assume that $E_{*}$ is asymptotically stable when $r=0$. Then the following statements hold:

1. If $1-\lambda c>0$, then $\mu\left(\mathcal{B}\left(E_{*}\right)_{r=0}\right) \leq \mu\left(\mathcal{B}\left(E_{*}\right)_{r=1}\right)$, where

$$
\mathcal{B}\left(E_{*}\right)_{r=1}:=\mathbb{R}_{+}^{2}, \quad \mathcal{B}\left(E_{*}\right)_{r=0} \subseteq \mathbb{R}_{+}^{2} \backslash \cup_{n=0}^{+\infty} T_{2}^{-n}\left(\mathcal{B}_{2}\right)
$$

2. If $0>1-\lambda c>-1$, and

$$
\frac{\lambda}{c \lambda-1} \frac{N-1}{N^{2}} \geq \frac{2-\alpha+\alpha N}{\alpha c N^{2}}
$$

then $\mu\left(\mathcal{B}\left(E_{*}\right)_{r=0}\right) \leq \mu\left(\mathcal{B}\left(E_{*}\right)_{r=1}\right)$, where

$$
\mathcal{B}\left(E_{*}\right)_{r=1}:=\mathbb{R}_{+}^{2} \backslash \mathcal{B}_{1} \quad \text { and } \quad \mathcal{B}\left(E_{*}\right)_{r=0} \subseteq \mathbb{R}_{+}^{2} \backslash \cup_{n=0}^{+\infty} T_{2}^{-n}\left(\mathcal{B}_{2} \cup \mathcal{B}_{3}\right)
$$

3. If $-1>1-\lambda c$, then $\mu\left(\mathcal{B}\left(E_{*}\right)_{r=1}\right)=0 \leq \mu\left(\mathcal{B}\left(E_{*}\right)_{r=0}\right)$, where

$$
\mathcal{B}\left(E_{*}\right)_{r=1}=E_{*} \quad \text { and } \quad \mathcal{B}\left(E_{*}\right)_{r=0} \subseteq \mathbb{R}_{+}^{2} \backslash \cup_{n=0}^{+\infty} T_{2}^{-n}\left(\mathcal{B}_{2} \cup \mathcal{B}_{3}\right)
$$


Proof. First of all, recall that, by Proposition 3 , conditions $2 \leq N<1+\frac{4}{\alpha}$ and $\lambda<\frac{N}{c}$ guarantee that $E_{*}$ is asymptotically stable when $r=0$. With $r=1$ (all G-agents), the map (17) becomes

$$
T_{2, r=1}:\left\{\begin{array}{l}
x(t+1)=\max \left\{0, x(t)+\lambda x(t)\left(\frac{(N-1) x(t)}{x(t)^{2} N^{2}}-c\right)\right\}=\max \left\{0, f_{x}\right\} \\
y(t+1)=\max \left\{0,(1-\alpha) y(t)+\frac{\alpha}{2}[y(t)+N x(t)(1-c N x(t))]\right\}=\max \left\{0, f_{y}\right\}
\end{array}\right.
$$

where the first component is a master equation, i.e. a one-dimensional difference equation uncoupled from the second one, whereas the second component is a slave equation, because it depends also on the first variable ${ }^{15}$ The properties of the one-dimensional map $f_{x}$, which is a (piecewise-)linear map, strongly influence the dynamics of the whole system. The Nash equilibrium $E_{*}=\left(\frac{N-1}{c N^{2}}, \frac{N-1}{c N^{2}}\right)$ is a fixed point of 29) and the asymptotic dynamics depend on the initial conditions of the system (29). Specifically, let us consider the following subset of $\mathbb{R}_{+}^{2}$, which represents the points of $\mathbb{R}_{+}^{2}$ that are mapped by 29$]$ into $(0, y)$ in one iteration:

$$
\begin{aligned}
\mathcal{B}_{1} & :=\left\{(x, y) \in \mathbb{R}_{+}^{2} \mid x(1-\lambda c)+\frac{\lambda(N-1)}{N^{2}} \leq 0\right\} \\
& =\left\{(x, y) \in \mathbb{R}_{+}^{2} \mid x \geq \frac{\lambda}{c \lambda-1} \frac{N-1}{N^{2}}\right\}
\end{aligned}
$$

It is clear that any trajectory starting with initial condition $(x, y) \in \mathcal{B}_{1}$ does not converge to $E_{*}$. Having assumed that $E_{*}$ is asymptotically stable and being the only attractor in the invariant region $\mathbb{R}_{+}^{2} \backslash \cup_{n=0}^{+\infty}$ $T_{2}^{-n}\left(\mathcal{B}_{1}\right)$, it follows that $\mathcal{B}\left(E_{*}\right)_{r=1}:=\mathbb{R}_{+}^{2} \backslash \cup_{n=0}^{+\infty} T_{2}^{-n}\left(\mathcal{B}_{1}\right)$.

With $r=0$, (all LMA-agents), the map (17) becomes

$$
T_{2, r=0}:\left\{\begin{array}{l}
x(t+1)=\max \left\{0, x(t)+\lambda x(t)\left(\frac{(N-1) y(t)}{[x(t)+(N-1) y(t)]^{2}}-c\right)\right\}=\max \left\{0, f_{x}\right\} \\
y(t+1)=\max \left\{0,(1-\alpha) y(t)+\frac{\alpha}{2}[y(t)+N y(t)(1-c N y(t))]\right\}=\max \left\{0, f_{y}\right\}
\end{array}\right.
$$

where the second component is the master equation (uncoupled from the first one), while the first component depends also on the second variable (slave equation). The properties of the one-dimensional map $f_{y}$, which is a quadratic map conjugates to the well known logistic map, strongly influence the dynamics of the system. The fixed point of (31) is again the Nash equilibrium $E_{*}=\left(\frac{N-1}{c N^{2}}, \frac{N-1}{c N^{2}}\right)$, and the asymptotic dynamics depends on the initial conditions. Specifically, let us consider the following two subregions of $\mathbb{R}_{+}^{2}$ :

$$
\begin{aligned}
\mathcal{B}_{2} & :=\left\{(x, y) \in \mathbb{R}_{+}^{2} \mid f_{y} \leq 0\right\} \\
& =\left\{(x, y) \in \mathbb{R}_{+}^{2} \mid y \geq \frac{2-\alpha+\alpha N}{\alpha c N^{2}}\right\} \\
\mathcal{B}_{3} & :=\left\{(x, y) \in \mathbb{R}_{+}^{2} \mid f_{x} \leq 0\right\} \\
& =\left\{(x, y) \in \mathbb{R}_{+}^{2} \mid x^{2}+(N-1)^{2} y^{2}+(N-1) x y-\frac{\lambda(N-1)}{\lambda c-1} y \leq 0\right\}
\end{aligned}
$$

It is clear that any trajectory starting in $(x, y) \in \mathcal{B}_{2} \cup \mathcal{B}_{3}$ will never converge to $E_{*}$, while, assuming $E_{*}$ asymptotically stable, its basin of attraction is given by $\mathcal{B}\left(E_{*}\right)_{r=0} \subseteq \mathbb{R}_{+}^{2} \backslash \cup_{n=0}^{+\infty} T_{2}^{-n}\left(\mathcal{B}_{2} \cup \mathcal{B}_{3}\right)$.

Considering $r=0$, it is trivial to prove that the set $\mathcal{B}_{3}$ is empty for $1-\lambda c>0$, while any trajectory starting in $\mathcal{B}_{2}$ is lead in the subregion (axis) $y=0$, where the dynamics is undefined and so the oligopoly is infeasible. On the contrary, trajectories starting in $\mathbb{R}_{+}^{2} \backslash \cup_{n=0}^{+\infty} T_{2}^{-n}\left(\mathcal{B}_{2}\right)$ converge either to $E_{*}$ (assuming $E_{*}$ asymptotically stable) or to some attractor contained in the region. Moreover, considering $r=1$, it is trivial to prove that $\mathcal{B}_{1}$ is empty for $1-\lambda c>0$. It follows that $\mathcal{B}\left(E_{*}\right)_{r=1}:=\mathbb{R}_{+}^{2}$. Noting that $\mathbb{R}_{+}^{2} \backslash \cup_{n=0}^{+\infty} T_{2}^{-n}\left(\mathcal{B}_{2}\right) \subseteq \mathcal{B}\left(E_{*}\right)_{r=1}:=\mathbb{R}_{+}^{2}$, the first part of the proposition follows.

Considering $r=0$, it is easy to see that for $\lambda c>1$ the set $\mathcal{B}_{1}$ is not empty and preimages in $\mathbb{R}_{+}^{2}$ of the $x=0$ and $y=0$ axis exist as sketched in Figure 4. It follows that $\mathcal{B}\left(E_{*}\right)_{r=0} \subseteq \mathbb{R}_{+}^{2} \backslash \cup_{n=0}^{+\infty} T_{2}^{-n}\left(\mathcal{B}_{2} \cup \mathcal{B}_{3}\right)$.

\footnotetext{
${ }^{15}$ Map 29, as well as map 31 below, are two triangular maps. For more details on these maps see e.g. [29, [30] and [31] .
} 
Moreover, considering $r=1$, if $0>1-\lambda c>-1$ it is straightforward to prove that every trajectory starting in $\mathcal{B}_{1}$ converges to the $x=0$ axis where the map is undefined as it is a set of focal points whereas each trajectory starting in $\mathbb{R}_{+}^{2} \backslash \mathcal{B}_{1}$ converges to the asymptotically stable Nash equilibrium $E_{*}$, which is also the unique attractor in the region. This can be proved easily noting that the map $f_{x}$ is linear in $\mathbb{R}_{+}^{2} \backslash \mathcal{B}_{1}$, so it follows that $\mathcal{B}\left(E_{*}\right)_{r=1}:=\mathbb{R}_{+}^{2} \backslash \mathcal{B}_{1}$ and $\cup_{n=0}^{+\infty} T_{2}^{-n}\left(\mathcal{B}_{1}\right)=\mathcal{B}_{1}$. If condition 26 holds, it is easy to see that the area of $\mathcal{B}_{1}$ is smaller then the area of $\mathcal{B}_{2}$. Thus the second part of the proposition follows.

Considering $r=1$, if $1-\lambda c<-1$, then the Nash equilibrium $E_{*}$ is unstable and the first recurrence of $T_{2, r=1}$, i.e. $\max \left\{0, f_{x}\right\}$ in $(29)$, is a linear function as long as $x(t)>0$. It follows that each trajectory of $f_{x}$ starting in $\mathbb{R}_{+}^{2} \backslash E_{*}$ diverges to $-\infty$ and so every trajectory of $T_{2}$ starting in $\mathbb{R}_{+}^{2} \backslash E_{*}$ converges to the focal point $(0,0)$. The third point of the proposition follows. QED

Proposition 4 provides important insights about the robustness of the asymptotic stability of the Nash equilibrium, measured by the area of the basin of attraction of the equilibrium itself, when all the firms involved in the oligopoly adopt the same heuristic, i.e. either the $\mathrm{G}$ or the LMA rule. In particular, the three cases discussed in Proposition 4 are depicted in Figure 4 while in Figure 5 regions $\mathcal{B}_{2}$ and $\mathcal{B}_{3}$ are depicted for $r=0$, together with the basin of attraction $\mathcal{B}\left(E_{*}\right)_{r=0}$. The picture shows the presence of lobes, which are typical when there are focal points, as it is the point $(0,0)$ for map $T_{2}$, see e.g. [25], 32] and [33] for details.

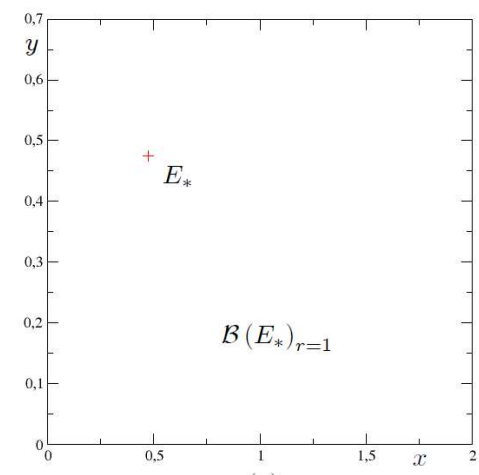

(a)

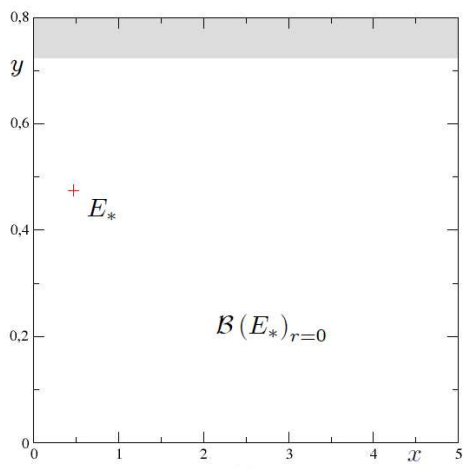

(d)

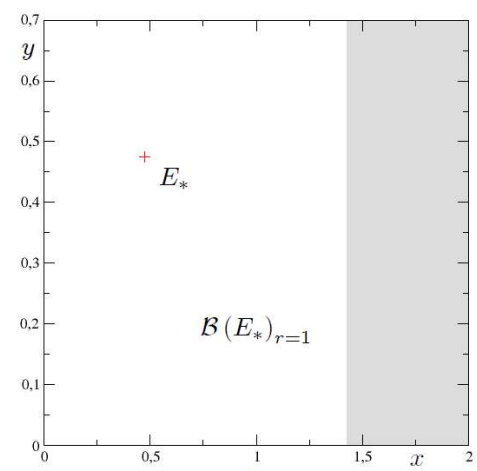

(b)

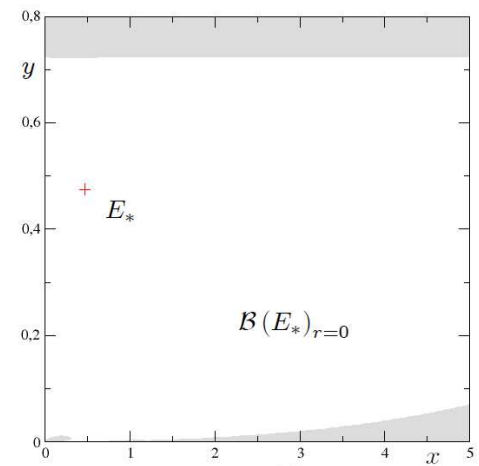

(e)

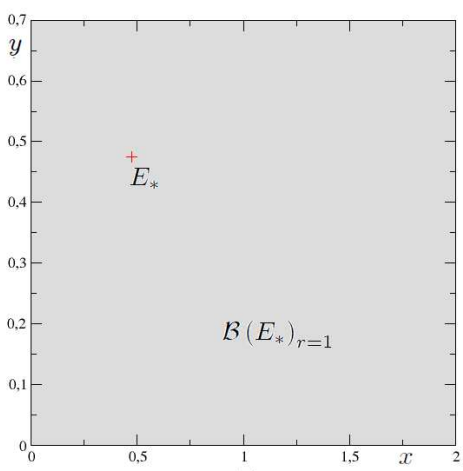

(c)

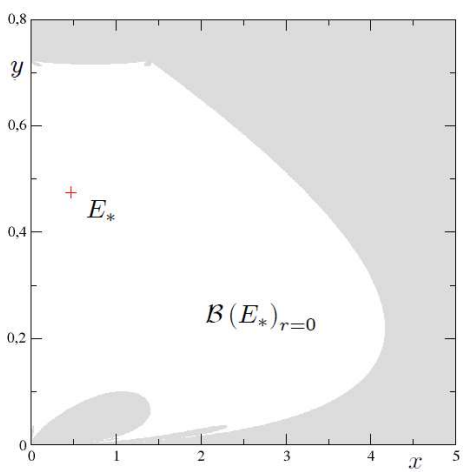

(f)

Figure 4: Basins of attraction $\mathcal{B}\left(E_{*}\right)_{r=1}$ (first row) and $\mathcal{B}\left(E_{*}\right)_{r=0}$ (second row) depicted as white regions. Regions of infeasible trajectories are in gray. Cases of Proposition 4 are represented from the left to the right: $\lambda c<1$ with $\lambda=1$ and $c=0.1$ (first column), $1<\lambda c<2$ with $\lambda=15$ and $c=0.1$ (second column) and $\lambda c>2$ with $\lambda=25$ and $c=0.1$ (third column). Parameters $N=20, \alpha=0.2$.

The economic intuition behind the results of this analysis is synthesized in the following remark.

Remark. Consider the dynamical system $T_{2}$ in (17) and its non-trivial Nash equilibrium $E_{*}$ in (18). As long as the conditions for the asymptotic stability of the Nash equilibrium are ensured for the oligopoly with 


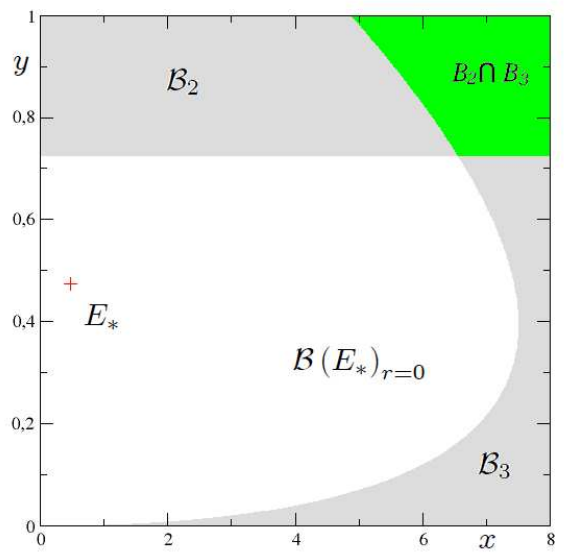

(a)

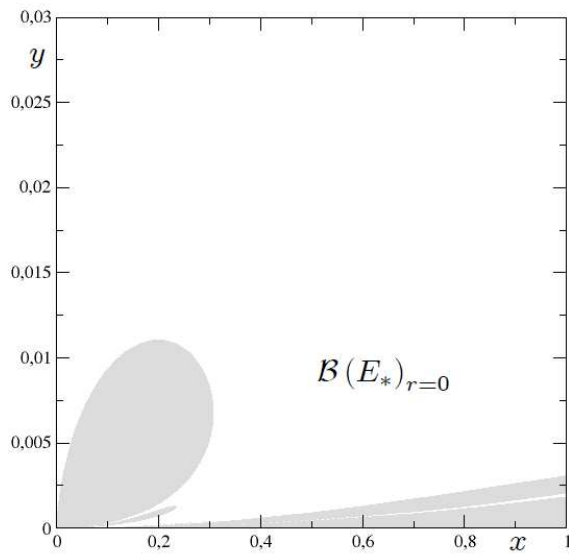

(b)

Figure 5: Panel (a) the white region is $\mathcal{B}\left(E_{*}\right)_{r=0} \subseteq \mathbb{R}_{+}^{2} \backslash \cup_{n=0}^{+\infty} T_{2}^{-n}\left(\mathcal{B}_{2} \cup \mathcal{B}_{3}\right)$. Points in $\mathcal{B}_{2} \cap \mathcal{B}_{3}$ (green region) are mapped in one iteration in $(0,0)$. Panel (b) zoom of $\mathcal{B}\left(E_{*}\right)_{r=0}$ near the origin, which is a focal point of the map. The regions of infeasible trajectories, i.e. $\mathcal{B}_{2}$ and $\mathcal{B}_{3}$, and their preimages are in gray. Parameters: $N=20, c=0.1, \lambda=15, \alpha=0.2$.

all G-firms, i.e. $\lambda c<2$, and the following inequality holds

$$
\frac{\lambda}{|1-\lambda c|} \geq \frac{2-\alpha+\alpha N}{(N-1) \alpha c}
$$

then $\mathcal{B}\left(E_{*}\right)$, the basin of attraction of the Nash equilibrium $E_{*}$, with all G-firms is at least as large as the corresponding basin in the case of all LMA-firms. On the contrary, when the Nash equilibrium with all G-firms is unstable, i.e. $\lambda c>2$, the presence of LMA-firms is necessary, but not sufficient, to ensure the feasibility of the oligopoly.

In order to provide some insights about the dimensions of the basins of attraction of the Nash equilibrium $E_{*}$ also with a mixture of G-firms and LMA-firms, i.e. $r \in(0,1)$, two cases are shown in Figures 6 and 7 In Figure 6 we have the first case discussed in Proposition 4, i.e. $\lambda c<1$. In particular, for $r=1$ and $r=0$, we have the same cases of Panels (a) and (d) of Figure 4. respectively. Note that for $r=0.5$, i.e. half of the firms adopt the LMA rule and the other half the G-rule, the basin of attraction of $E_{*}$ increases considerably with respect to the case $r=0$, and it becomes similar to the basin obtained with $r=1$. In Figure 7, the case $1<\lambda c<2$ is considered. In particular, for $r=0$ and $r=1$, we have the same cases of Panels (b) and (d) of Figure 4, respectively. In this case, the presence of a very small fraction of G-firms in the oligopoly $\left(0,1 \%\right.$ in the example) considerably enlarges $\mathcal{B}\left(E_{*}\right)$, the basin of attraction of the Nash equilibrium, so that the oligopoly acquires stability in the state space.

\subsection{Dynamic analysis of the evolutionary model}

We begin the analysis of the dynamical system $T$ in (15) by studying the existence and local stability of equilibria. This question is quite simple whenever the Nash equilibria of the two-dimensional restrictions to the invariant planes $r=0$ or $r=1$ are stable.

We can reformulate Proposition 1 and Corollary 2 with the specific map (15) in a unique proposition:

Proposition 5. Consider the dynamical system $T$ in 115 .

- If $K_{G}=K_{L}$, then a continuum of equilibrium points $E_{*}$ exists along the segment $E=\left(\frac{N-1}{c N^{2}}, \frac{N-1}{c N^{2}}, r\right)$, with $r \in[0,1]$. These equilibria are stable as long as the stability conditions for the Nash equilibrium stated in Proposition 3 are satisfied. One fixed point in $E$ undergoes a codimension-two bifurcation changing $K_{G}-K_{L}$ with an associated eigenvalue equal to 1 and another one equal to -1 , with the 


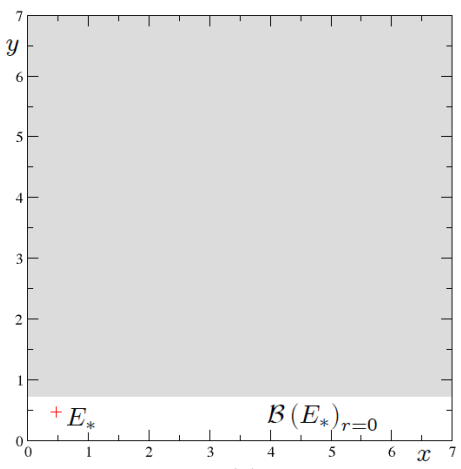

(a)

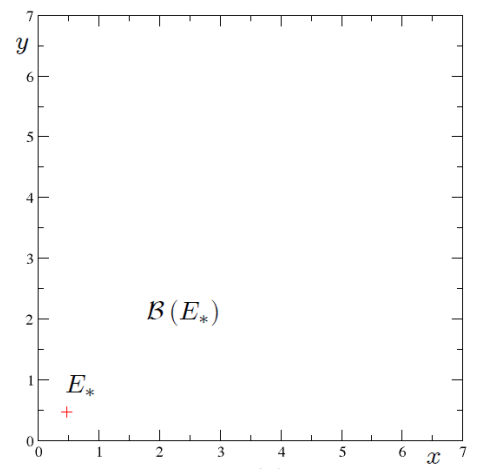

(b)

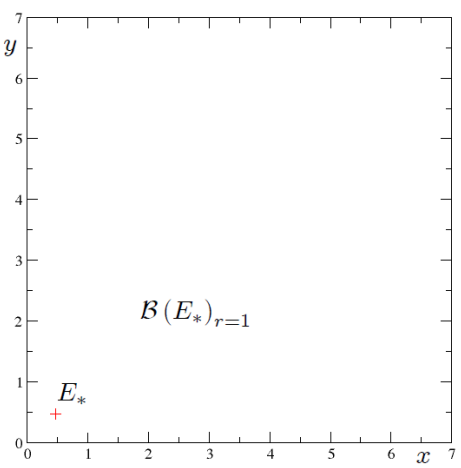

(c)

Figure 6: Basins of attraction of $E_{*}$ (white region), for different values of $r$ : panel (a) $r=0$, panel (b) $r=0.5$ and panel (c) $r=1$. Region of infeasible trajectories in gray. Parameters: $\lambda=1, N=20, c=0.1, \alpha=0.2$.

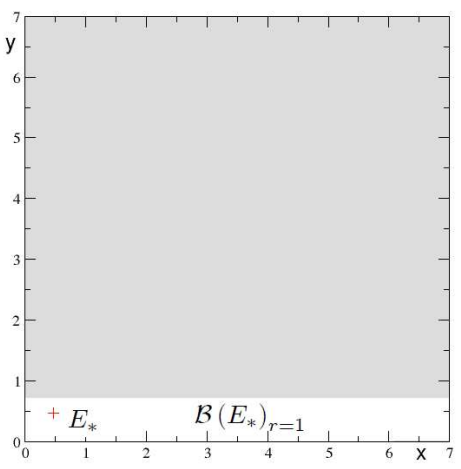

(a)

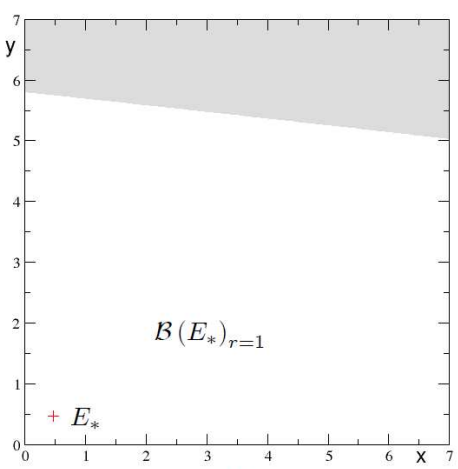

(b)

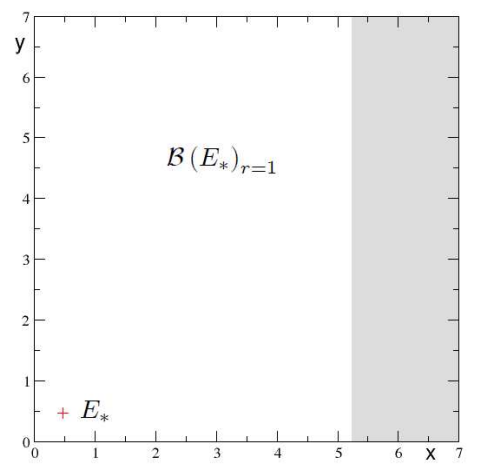

(c)

Figure 7: Basins of attraction of the Nash-equilibrium $E_{*}$ (white region) for different values of $r$ : panel (a) $r=0$, panel (b) $r=0.001$ and panel (c) $r=1$. Region of infeasible trajectories in gray. Parameters: $\lambda=15, N=20, c=0.1, \alpha=0.2$.

possible creation of a stable 2-cycle $\{(\underline{x}, \underline{y}, \underline{r}),(\bar{x}, \bar{y}, \bar{r})\}$. The point in $E$ that undergoes the codimensiontwo bifurcation is the one such that $\left(x^{+}, y^{+}, r^{+}\right)=(\underline{x}, \underline{y}, \underline{r})=(\bar{x}, \bar{y}, \bar{r})$ and $\lambda=\lambda^{*}\left(\alpha, r^{+}\right)$, where

$$
\lambda^{*}\left(\alpha, r^{+}\right)=\frac{2 N\left(\alpha\left(1-2 r^{+}-N\left(1-r^{+}\right)\right)+4\right)}{c\left(r^{+}(\alpha+4)(N-2)-2 \alpha(N-1)+8\right)}
$$

- If $K_{G} \neq K_{L}$, then only the two extremum points of the segment $E$ are equilibria, namely

$$
E_{0}=\left(\frac{N-1}{c N^{2}}, \frac{N-1}{c N^{2}}, 0\right) \quad \text { and } \quad E_{1}=\left(\frac{N-1}{c N^{2}}, \frac{N-1}{c N^{2}}, 1\right)
$$

in which all agents adopt the same strategy, which is LMA or G respectively:

- If $K_{L}<K_{G}$, then equilibrium $E_{0}$ is stable for $2 \leq N<1+\frac{4}{\alpha}$ and $\lambda<\frac{N}{c}$, and it loses stability through a period doubling bifurcation at $N=1+\frac{4}{\alpha}$ given $\lambda<\frac{N}{c}$. $E_{1}$ is always unstable.

- If $K_{L}>K_{G}$, then equilibrium $E_{1}$ is stable for $\lambda<\frac{2}{c}$ and loses stability for $\lambda>\frac{2}{c}$. At $\lambda=\frac{2}{c}$ no bifurcation occurs. $E_{0}$ is always unstable.

Proof. The proof is a direct application of Proposition 1, Corollary 2 and Proposition 3. 
Starting from the previous investigation of the dynamics of the model on the invariant subspaces $r=0$ and $r=1$, Proposition 5 describes, for the case $K_{G} \neq K_{L}$, the local asymptotic stability of the two Nash equilibria $E_{0}$ and $E_{1}$, which are located on the invariant planes $r=0$ and $r=1$ respectively. For the threedimensional model, the main question related to these equilibria concerns their local transverse stability. When an equilibrium on an invariant plane (either $r=0$ or $r=1$ ) is locally asymptotically stable for the bidimensional map (17) then it obviously attracts sufficiently nearby trajectories of the plane, i.e. it is stable with respect to perturbations along the plane. Transverse stability occurs when an attractor on the plane is also an attractor for the complete map (15), i.e. if it is stable with respect to perturbations that are transverse to the plane; in particular, these perturbations have initial conditions $(x(0), y(0), r(0))$ with $r(0) \in(0,1)$. In this case, the basin of attraction of the attractor on the plane has positive measure, i.e. the basin has positive volume, being a subset of $\mathbb{R}^{3}$.

For the sake of clarification, it is worth pointing out that the local transverse instability of one of the two equilibria, be it $E_{0}$ or $E_{1}$, does not exclude that they may also have a basin of attraction of positive Lebesgue measure in $\mathbb{R} \times \mathbb{R} \times[0,1]$. In other words, the Nash equilibria $E_{0}$ and $E_{1}$ might be Milnor (or weak) attractors, see [34] and [32. To have this, it is sufficient that a Nash equilibrium that belongs to an invariant plane is: 1) stable along the plane and 2) its basin of attraction on the plane intersects a set of points (with non-zero area) that are transversely stable. These points in the plane, which do not include the Nash equilibrium on the plane as we have assumed that it is transversely unstable, attract some trajectories with initial conditions $(x(0), y(0), r(0))$ with $r(0) \in(0,1)$. Once these trajectories reach the invariant plane, they converge to the Nash equilibrium, since they are in its basin of attraction.

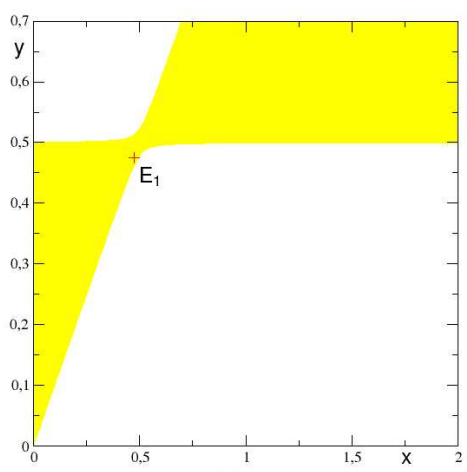

(a)

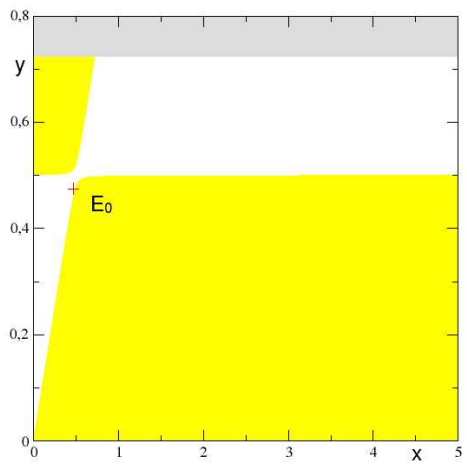

(d)

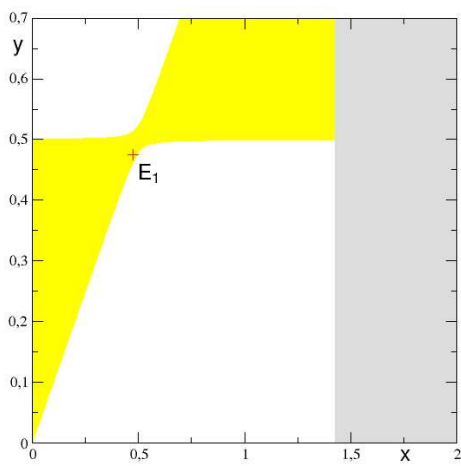

(b)

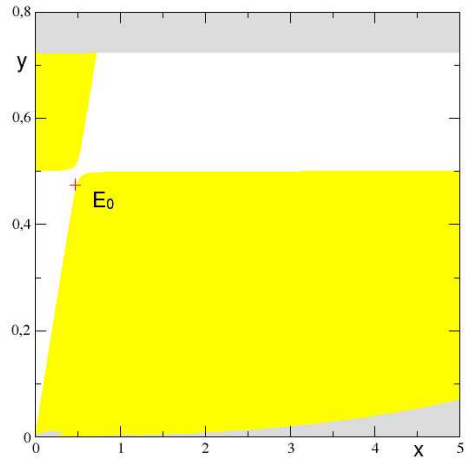

(e)

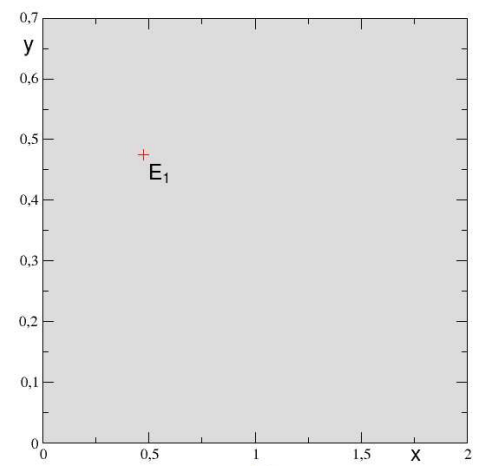

(c)

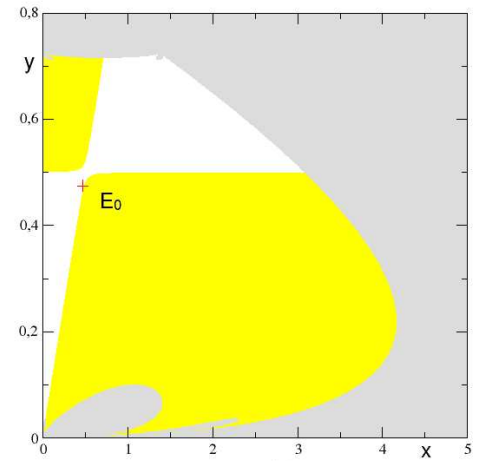

(f)

Figure 8: Regions of transversely unstable points (yellow region) and transversely attractive ones (white region) on invariant planes $r=1$ (first row) and $r=0$ (second row), when $K_{G}>K_{L}$. Regions of infeasible trajectories are in gray. Panels (a)-(d) $\lambda=1$. Panels (b)-(e) $\lambda=15$. Panels (c)-(e) $\lambda=25$. Parameters: $N=20, c=0.1, \beta=1, K_{G}=0.0001, K_{L}=0, \alpha=0.3$.

We illustrate this occurrence by numerical findings. Let us start considering the case $K_{G}>K_{L}$, i.e. agents have a propensity to adopt the LMA rule even in case the profits generated by the two heuristics are 
the same. In this case, as stated in Proposition 5, the Nash equilibrium $E_{0}$ is asymptotically stable while the Nash equilibrium $E_{1}$ is unstable. Nevertheless, in the basin of attraction of the Nash equilibrium $E_{1}$ are included points characterized by transverse attractivity (this set of points is represented by the white region in Figure 8 which attract trajectories coming from outside the invariant subspace. That is, the Nash equilibrium $E_{1}$ is a Milnor attractor. In Figure 8 , the situation is depicted both for the cases such that $E_{1}$ is stable, see panels (a) and (b), and unstable, see panel (c), on the invariant plane $r=1$. It is worth noticing that the dynamics of $T$ on the restrictions $r=0$ and $r=1$, is the same as the one depicted in Figure 4 and described in Proposition 4

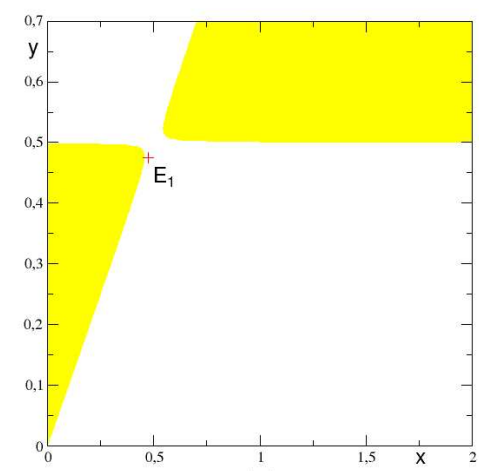

(a)

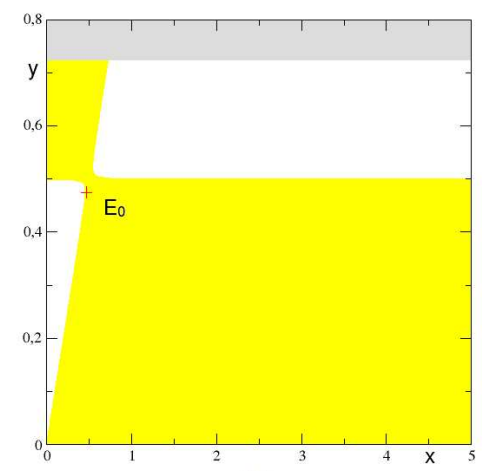

(d)

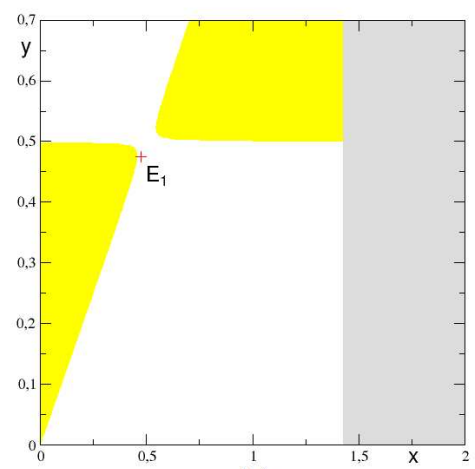

(b)

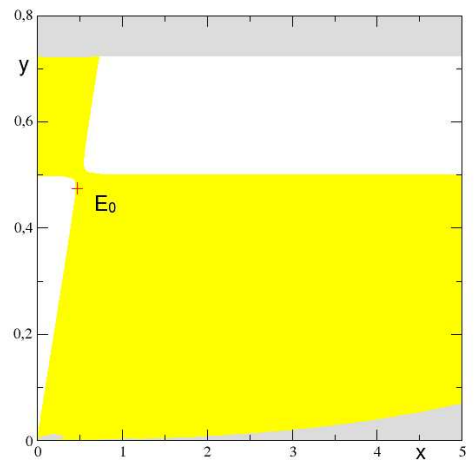

(e)

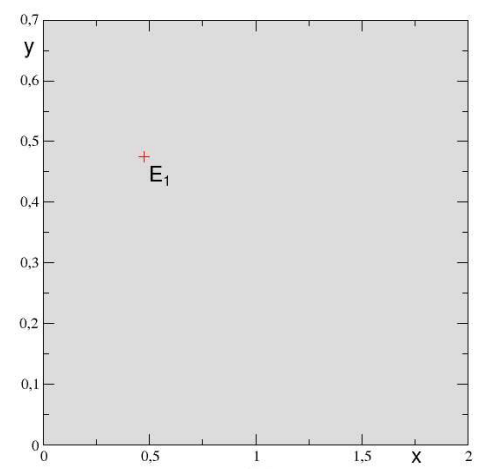

(c)

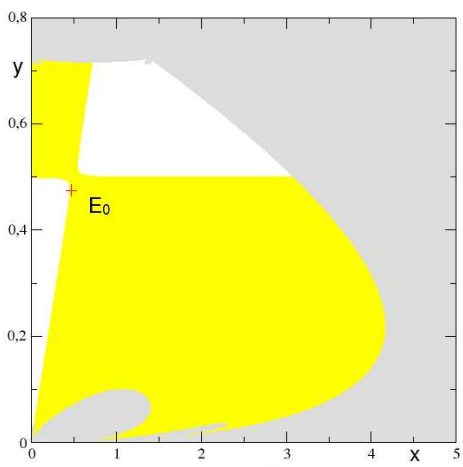

(f)

Figure 9: Regions of transversely unstable points (yellow region) and transversely attractive ones (white region) on invariant planes $r=1$ (first row) and $r=0$ (second row), when $K_{G}<K_{L}$. Regions of infeasible trajectories are in gray. Panels (a)-(d) $\lambda=1$. Panels (b)-(e) $\lambda=15$. Panels (c)-(e) $\lambda=25$. Parameters: $N=20, c=0.1, \beta=1, K_{G}=0, K_{L}=0.0001, \alpha=0.3$.

A similar situation occurs for cases with $K_{G}<K_{L}$, i.e. when agents have a propensity to adopt the G-rule even when profits generated by the two heuristics are equal. In this case, as stated in Proposition 5 , the Nash equilibrium $E_{1}$ is asymptotically stable while the Nash equilibrium $E_{0}$ is unstable. Nevertheless, the basin of attraction of the Nash equilibrium $E_{0}$ includes a set of points characterized by transverse attractivity (this set of points is represented by the white region in Figure 97. That is, the Nash equilibrium $E_{0}$ is a Milnor attractor. It is worth remembering that the structure of the basins of attraction of the two Nash equilibria $E_{0}$ and $E_{1}$ on $r=0$ and $r=1$ respectively is the same in Figures 8 and 9 as the only difference in the two cases regards the values of $K_{G}$ and $K_{L}$, which are two parameters that influence only the transverse stability. In particular, the particular shape of the basins of attraction, with the presence of lobes, is illustrated in details in the previous sections (see in particular Proposition 44, so it is not discussed here.

The analysis of transverse stability underlines another interesting dynamic property of the model. This occurs when, for $K_{G}>K_{L}$, the Nash equilibrium $E_{0}$ is unstable and the attractor on the invariant subspace $r=0$, generated by a sequence of period-doubling bifurcations, becomes transversely unstable. This situation 
is depicted in Figure $10^{16}$ From panel (a) of this figure we note that the attractor indicated by black dots has portions in both the white region and the yellow region. This indicates that along the attractor there are points that are transversely unstable (yellow region) and points that are transversely stable (white region). To provide a measure of the transverse attractivity of trajectory along the attractor, we compute the transverse Lyapunov exponents:

$$
\Lambda_{\perp}\left(T^{\infty}(x(0), y(0), r(0))\right)=\lim _{T \rightarrow \infty} \frac{1}{T} \sum_{i=0}^{T} \ln \left|v_{3}(x(i), y(i), r(i))\right|
$$

where $T^{\infty}(x(0), y(0), r(0)):=\left\{(x(i), y(i), r(i))=T^{i}(x(0), y(0), r(0)), i \geq 0\right\}$ is a generic trajectory along the attractor itself and

$$
v_{3}=\left.\frac{\partial r^{\prime}}{\partial r}\right|_{r=0}=e^{\beta \Delta \pi(x(i), y(i), 0)}
$$

is the eigenvalue of the map $T$ corresponding to the transversal direction. If $\Lambda_{\perp}\left(T^{\infty}(x(0), y(0), r(0))\right)<0$ holds, then average transversely stability of $T^{\infty}(x(0), y(0), r(0))$ is proved. In Figure 10 panel (b), we note that the transverse stability of the attractor is lost as $\alpha$ increases. When the attractor is transversely unstable, a situation of high uncertainty occurs for the evolutionary oligopoly game. Indeed, firms may fail to select a unique and common heuristic.

The analysis of the transverse stability of the attractors on the two invariant planes $r=0$ and $r=$ 1 underlines that the evolutionary selection of the heuristics by firms is a quite complicated nonlinear phenomenon and that a simple propensity of one rule to the other one does not necessary leads firms to adopt homogeneous behaviors.

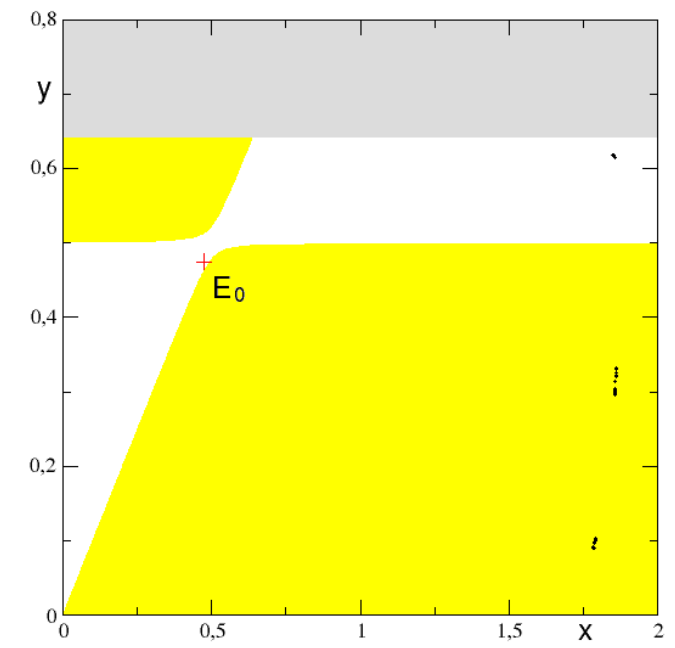

(a)

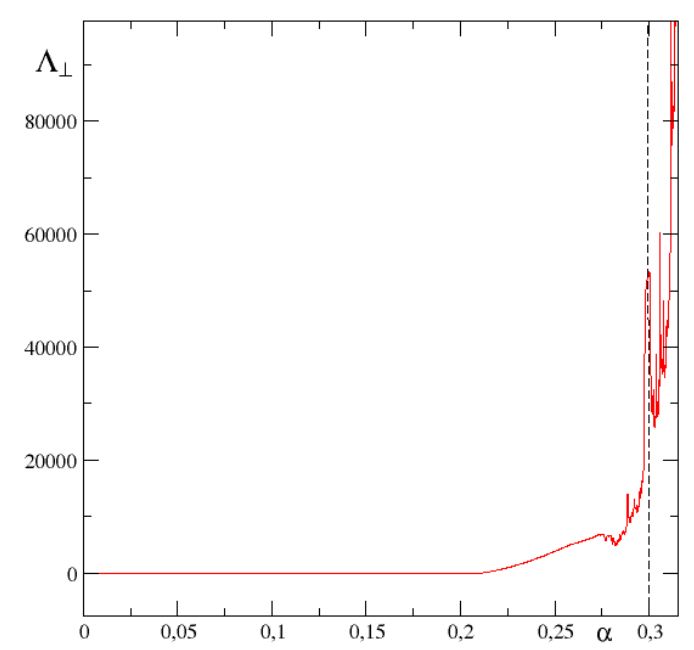

(b)

Figure 10: Panel (a) attractor on the invariant plane $r=0$. Its basin of attraction is in red. In yellow the set of points that are transversely unstable. Panel (b) Lyapunov exponent along the attractor of panel (a) as $\alpha$ varies in the range [0,0.3]. Parameters: $K_{G}=0.0001, K_{L}=0, N=20, \alpha=0.3, \lambda=1, N=20$.

This aspect is further confirmed by the possibility that an attractor not belonging to an invariant plane $(r=0$ or $r=1)$ exists. As indicated in Proposition 5 this attractor originates by a codimension-two bifurcation at $K_{G}=K_{L}$ from which a stable 2-cycle may appear. Moreover, this cycle may not be the

\footnotetext{
${ }^{16}$ Note that in this case $E_{0}$ is transversely stable as long as it is stable for the restriction of the map on the invariant plane $r=0$.
} 
unique stable attractor in $\mathbb{R} \times \mathbb{R} \times(0,1)$, as numerical evidence shows that other stable attractors could exist, see in particular the bifurcation diagram of Figure 11 .

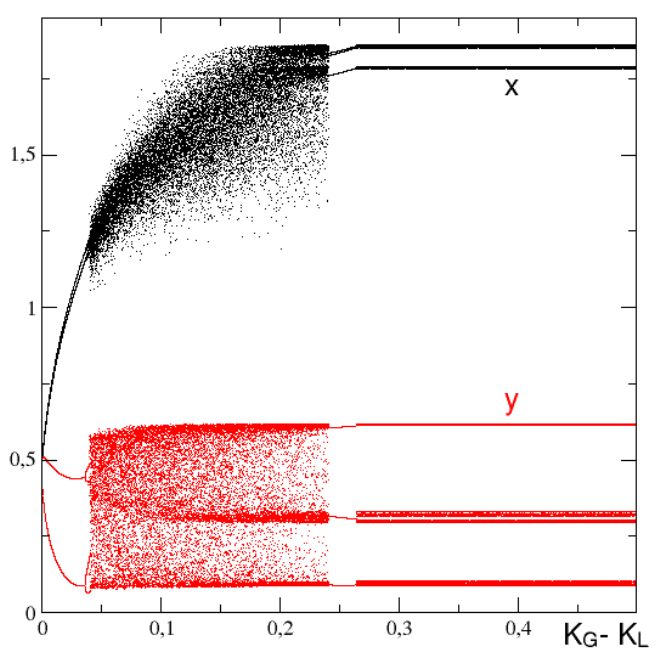

(a)

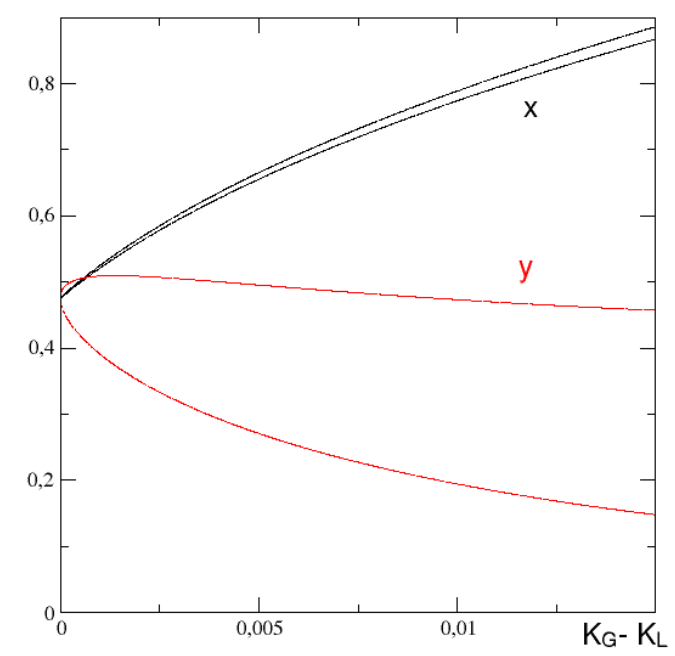

(c)

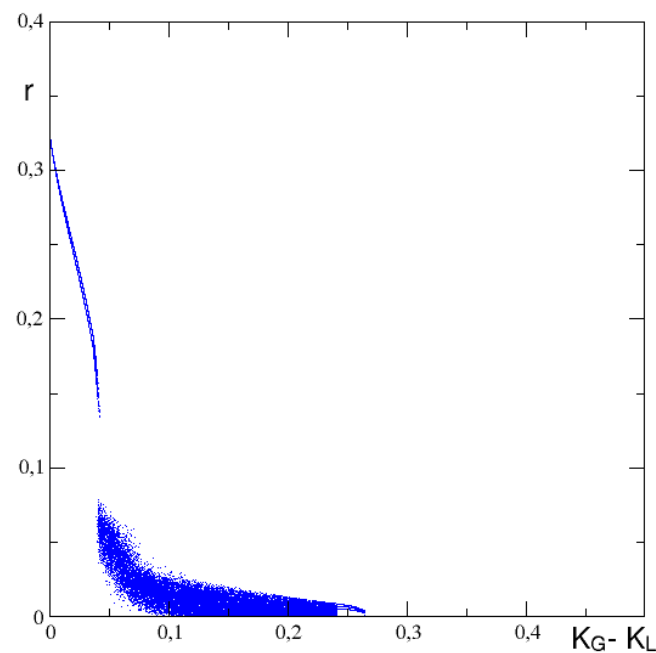

(b)

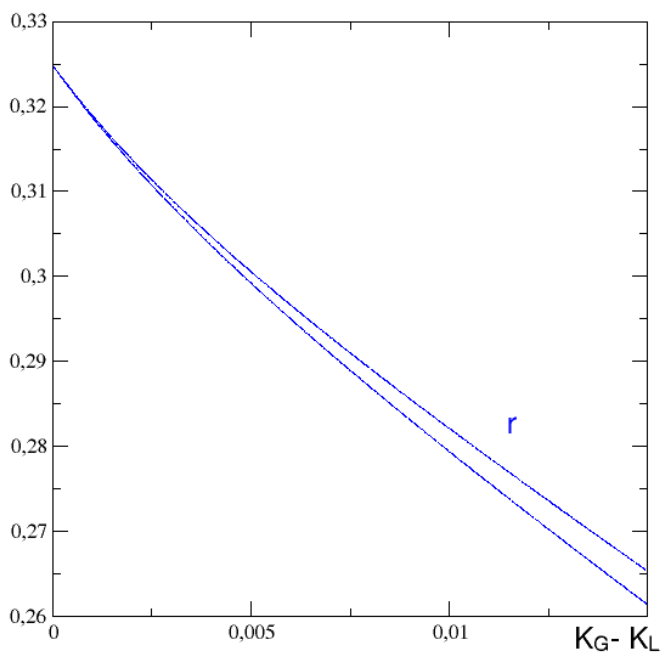

(d)

Figure 11: 1D-bifurcation diagram as $K_{G}-K_{L}$ varies on the range [0,0.5]. Panel (a), productions $x$ of a G-firm (black) and $y$ of a LMA-firm (red) as $K_{G}-K_{L}$ varies. Panel (b), dynamic of $r$ as $K_{G}-K_{L}$ varies. Panels (c) and (d) a zoom of the bifurcations diagrams on the upper line for $K_{G}-K_{L}$ close to zero. Parameters: $N=20, \beta=1, \alpha=0.3, \lambda=1$ and $c=0.1$.

The evolutionary oligopoly under analysis can exhibit further nonlinear phenomena originated by additional bifurcations that lead to complicated dynamics. For instance, the 2-cycle that is generated through the described codimension-two bifurcation (see e.g. Figure 12 represents two fixed points of the map $T^{2}$. Each of these two fixed points of $T^{2}$ can undergo a Neimark-Sacker bifurcation as the intensity of choice $\beta$ is increased. As a result, we detect the presence of an asymptotically stable attractor with $r \in(0,1)$ for $T$, see e.g. Figure 13. If the intensity of choice $\beta$ is further increased, the attractor takes a particular shape, see Figure 14 panel (d). The dynamics along this attractor is of particular interest from an economic point of view. Figure 14 presents a time series for the level of production. The dynamics of the quantity of production for LMA-firms and for G-firms follow a very strange pattern, which is characterized by long periods of almost 


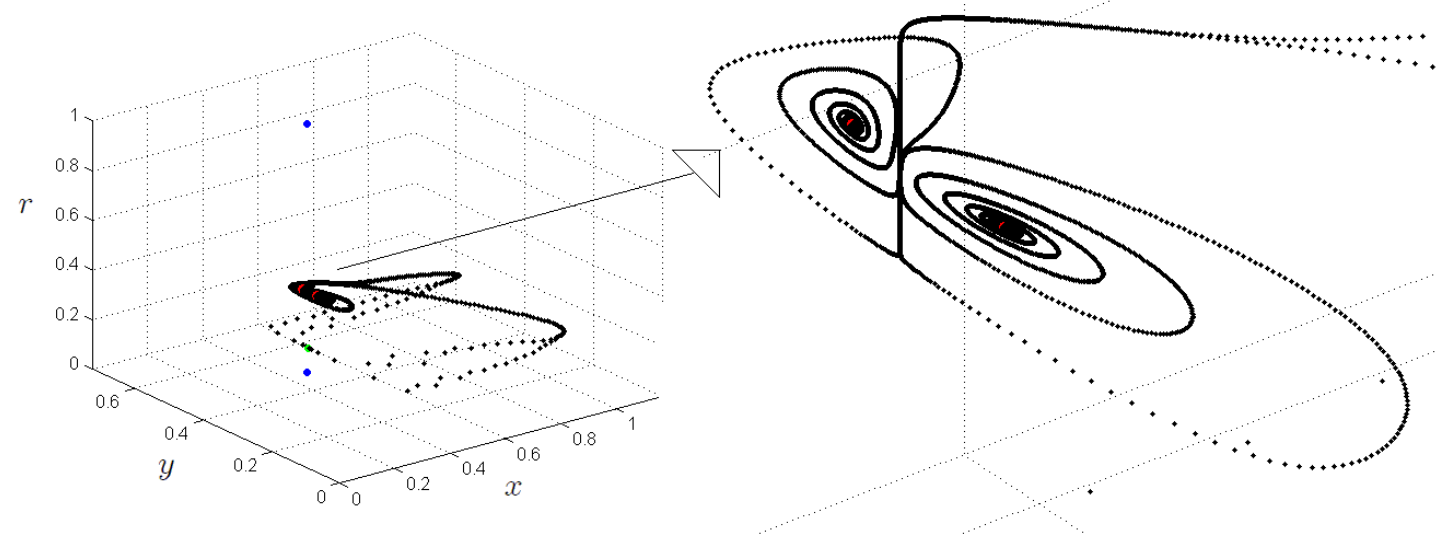

Figure 12: Trajectory for i.c. $\left(q^{*}-0.002, q^{*}+0.002,0.1\right)$. Parameters: $\lambda=1, N=20, c=0.1, \alpha=0.3, \beta=1, K_{y}=0$, $K_{x}=0.0001$. The Nash equilibria $E_{1}$ and $E_{0}$ are depicted in blue. The i.c. in green. The $\omega$-limit set is in red.

constant production levels - very close to the Nash quantity - that are interrupted by shorter periods of high production fluctuations. These dynamic scenarios shed some light on the high unpredictability of agents' behavior in the evolutionary oligopoly. Indeed, a long time period on which firms deliver almost constant productions, very close to the Nash equilibrium level, could lead an observer to think that the oligopoly is reaching a stationary equilibrium. Nevertheless, large bursts appear for short periods of times and at regular intervals to break the time series of constant productions.

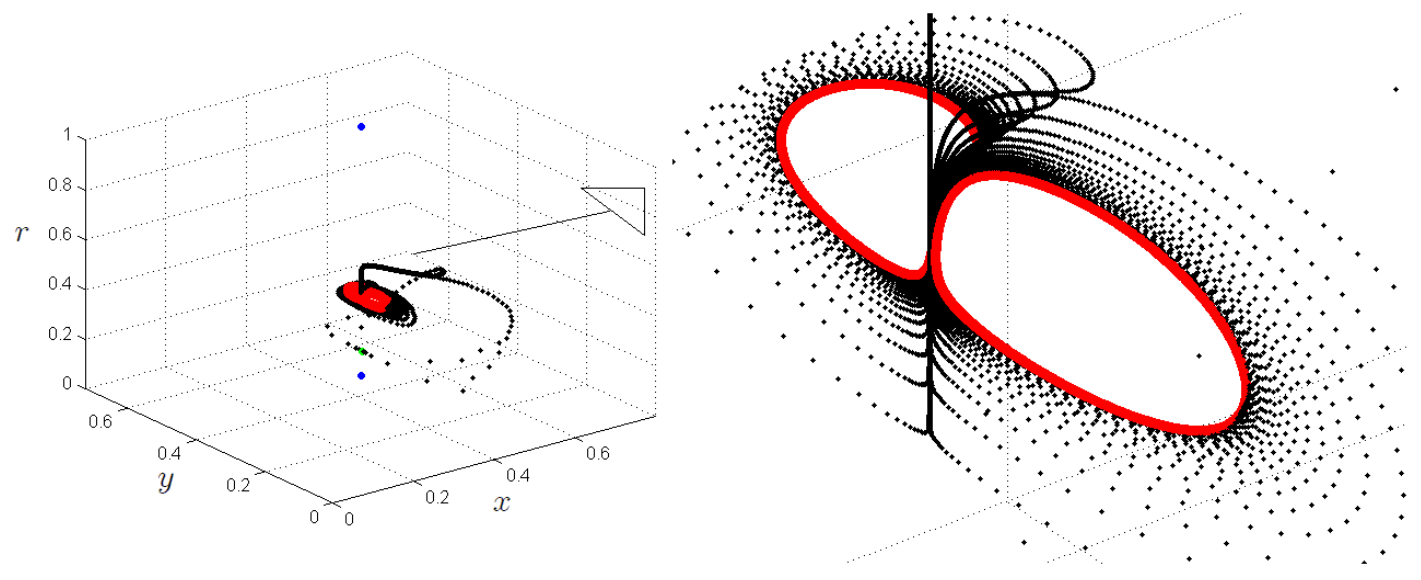

Figure 13: Trajectory for i.c. $\left(q^{*}-0.002, q^{*}+0.002,0.1\right)$. Parameters: $\lambda=1, N=20, c=0.1, \alpha=0.3, \beta=6, K_{y}=0$, $K_{x}=0.0001$. The Nash equilibria $E_{1}$ and $E_{0}$ are depicted in blue. The i.c. in green. The $\omega$-limit set is in red.

Another aspect is worth of being mentioned. The periodic and aperiodic attractors depicted in Figures 12,13 and 14 provide indication that it may be more profitable for firms to behave differently than similarly. Indeed, along these inner attractors the average level of production is lower than in the Nash equilibrium and this implies, given the profit function of the industry here assumed, that the average profit of the industry is higher than the profit with all firms at the Nash equilibrium. Specifically, we observe that the level of production of G-firms is always lower than the Nash equilibrium quantity and their profits are always higher than the one obtainable at the Nash equilibrium, see Figure 15 where we depicted the profit dynamics along the attractors of the previous examples. On the contrary, the levels of production of LMA-firms can oscillate around the Nash quantity and the profits can oscillate above and below the ones obtainable at the Nash equilibrium. However, relative to the dynamic scenario depicted in Figure 14, this occurs only for short intervals of time, while for most of the time both G-firms and LMA-firms experience higher profits along the 

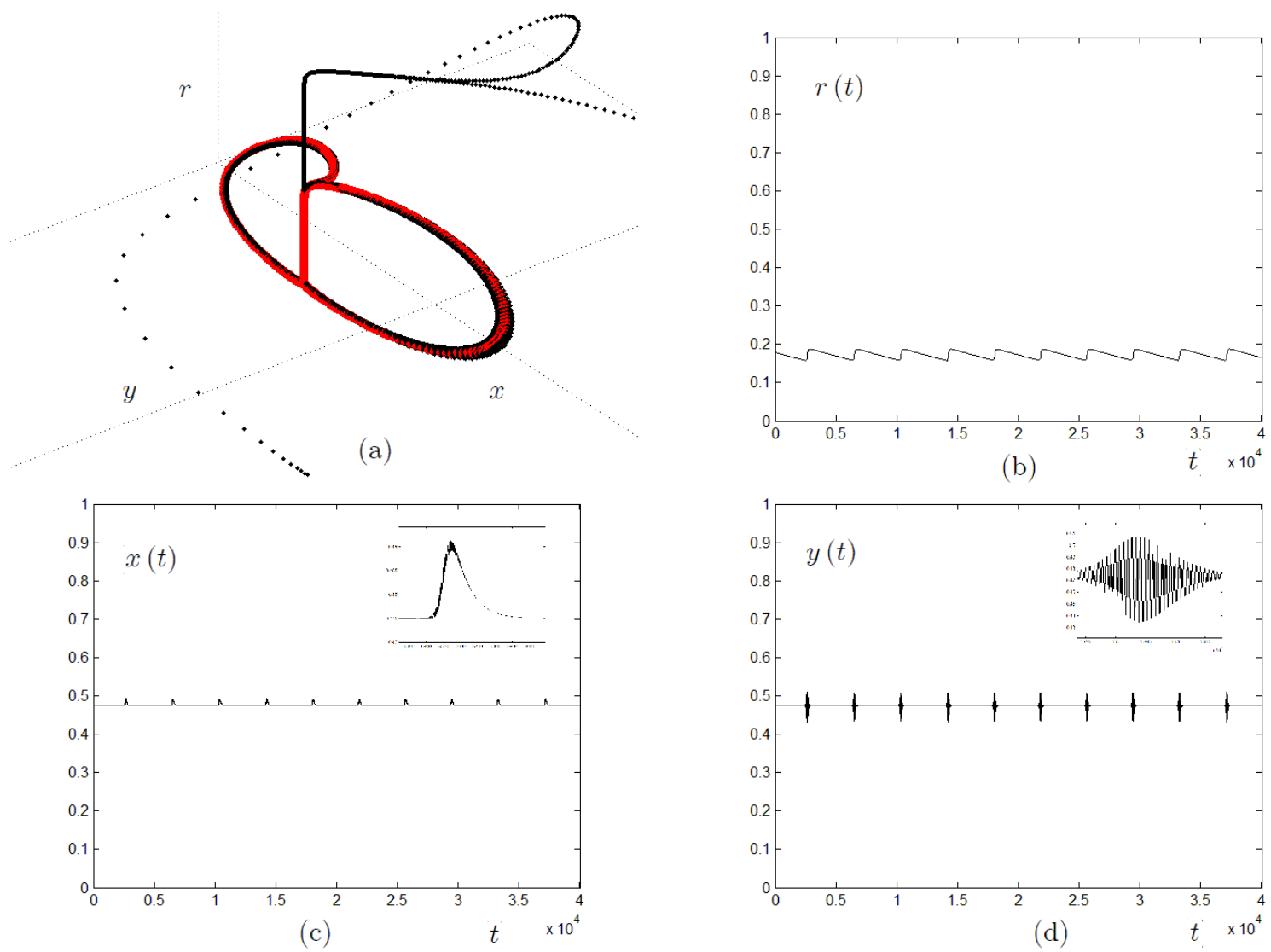

Figure 14: Panel (a) trajectory for i.c. $\left(q^{*}-0.002, q^{*}+0.002,0.1\right)$. The $\omega$-limit set is in red. Panels (b)-(d) time series of $r(t)$, $x(t)$ and $y(t)$. Parameters: $\lambda=1, N=20, c=0.1, \alpha=0.25, \beta=6, K_{y}=0, K_{x}=0.00001$.

inner attractor than at the Nash equilibrium. The economic explanation of this phenomenon is clear and based on the fact that for firms it is sufficient to produce less than the Nash quantity to increase their profits. This requires capability of coordination between firms, for example as if they would be able to constitute a cartel, which is not achievable within the simple evolutionary model proposed. However, a sufficient level of evolutionary competition (remember that inner attractors appear as the value of $\beta$ increases, parameter that measures the evolutionary pressure) between two boundedly-rational behavioral rules can sustain unexpected forms of coordinations that lead the industry as a whole and both groups of players as well to experience average levels of profits, which are higher than those obtainable at the Nash equilibrium. This observation leads to the conclusion that in some cases firms might prefer to be in a polymorphic rather than monomorphic state. Recapping, the heterogeneity in the firms' choice of the behavioral rules may lead to increments in the producer surplus (total profit of the industry), even though at the expenses of reducing the consumer surplus.

Besides the described phenomena, other form of complexity can arise as well. An example is depicted in Figure 16 where in panel (d) we observe a quite irregular dynamics of the fraction of G-firms that populates the evolutionary game as time evolves. In this case, a chaotic inner attractor exists.

To conclude the investigation on the dynamics of the evolutionary model, we can sum up by saying that asymptotic dynamics can exhibit evolutionary stable heterogeneity: the dynamics along the asymptotically stable attractors on $\mathbb{R} \times \mathbb{R} \times(0,1)$ indicate that heterogeneous behavior can be more profitable, in an evolutionary sense, than following a homogeneous strategy over time. Indeed, this heterogeneous behavior is the result of the evolutionary selection based on profits. The proposed analysis underlines also that a bias for playing one strategy does not necessarily imply that in the long run all the firms decide to adopt that strategy. More precisely, the propensity for playing one strategy does not exclude that firms select in the 
evolutionary process the other strategy in the long run. This crucially depends on several elements, but principally on the initial condition of the system. As a final note, we emphasize the possibility of complicated dynamics of the state variables generated by the evolutionary stable heterogeneities, which are particular interesting both from an economic and mathematical point of view.

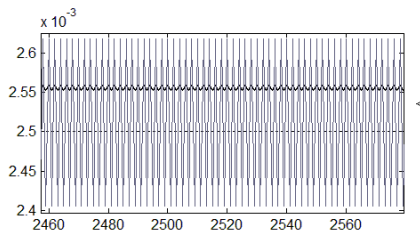

(a)

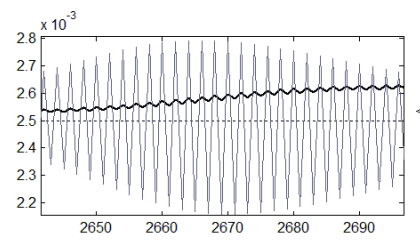

(d)

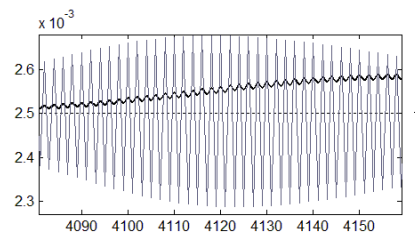

(g)

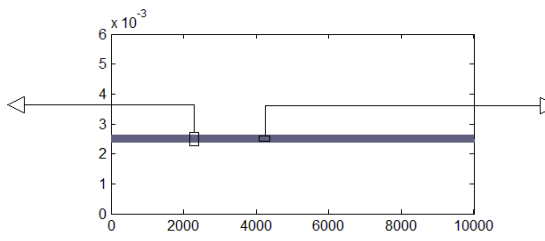

(b)

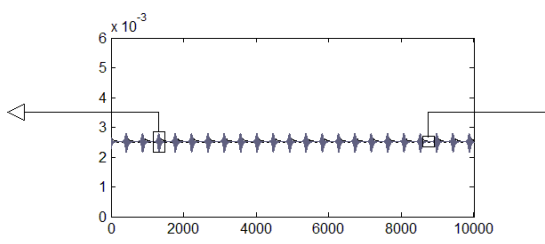

(e)

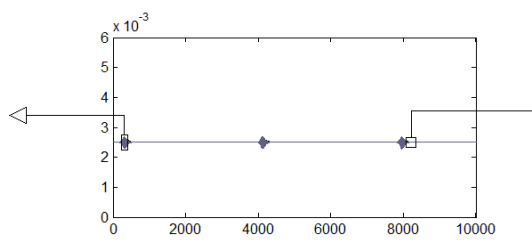

(h)

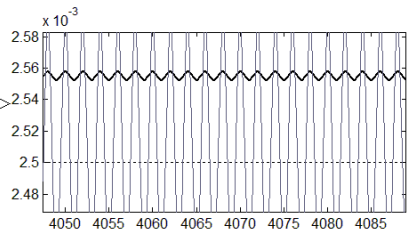

(c)

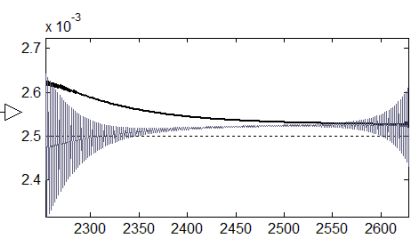

(f)

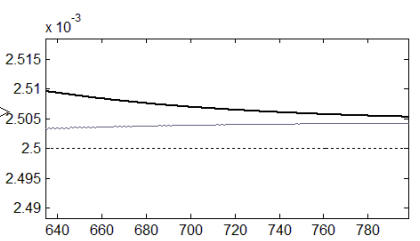

(i)

Figure 15: Dynamics of profits: Dotted line represents profits that firms would obtain at the Nash equilibrium, black solid line represents profits made by G-firms and gray solid line represents profits made by LMA-firms. First row, profit dynamics along the attractor depicted in Figure 12 Second row, profit dynamics along the attractor depicted in Figure 13 Third row, profit dynamics along the attractor depicted in Figure 14 Central column contains panels representing the profit dynamics along an attractor. Left and Right columns contains panels representing enlargements of some interesting parts of the profit dynamics.

\section{Conclusions}

In this paper, we considered an evolutionary oligopoly model where firms employ different heuristics for specifying their next period productions. After studying some general properties of the game, we explore the model with two particular behavioral rules, namely Local Monopolistic Approximation (LMA) and Gradient dynamics $(\mathrm{G})$, thus proposing a possible method for analyzing similar models. Despite its simple properties when convergence to the Nash equilibrium is achieved, the model is pretty rich from a dynamical point of view. First of all, we study the possible attractors and their basins on the invariant planes, which are planes where all agents adopt the same rule. These attractors can be transversely stable and constitute the long-run attractors of the evolutionary model. In addition, we show the existence of Milnor attractors on the invariant planes. Then, we analyze the existence of cyclic or chaotic attractors where both behavioral rules are adopted, thus providing examples of evolutionary stable heterogeneity, i.e. polymorphic population states where agents find useful to adopt both rules over time.

Several extensions of this model are worth being considered further. First of all, an interesting addition is to include memory in the fitness measures of the two strategies, following [35]. With respect to the economic structure, another interesting question, often overlooked by the existing literature, is to study how the elasticity of demand influences the overall properties of the system. We leave these questions to our research agenda.

\section{Acknowledgements}




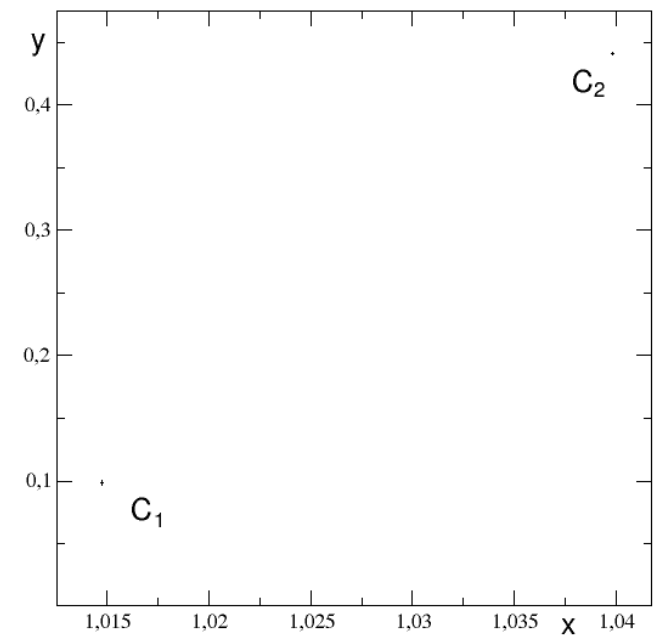

(a)

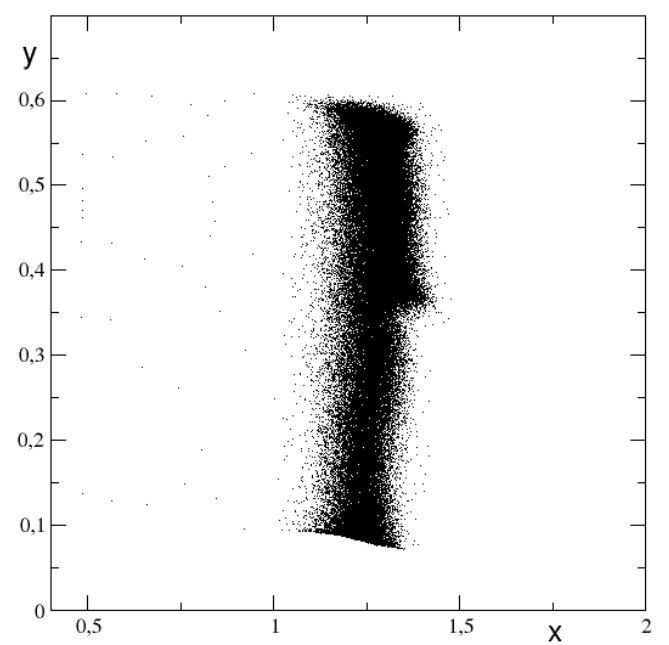

(c)

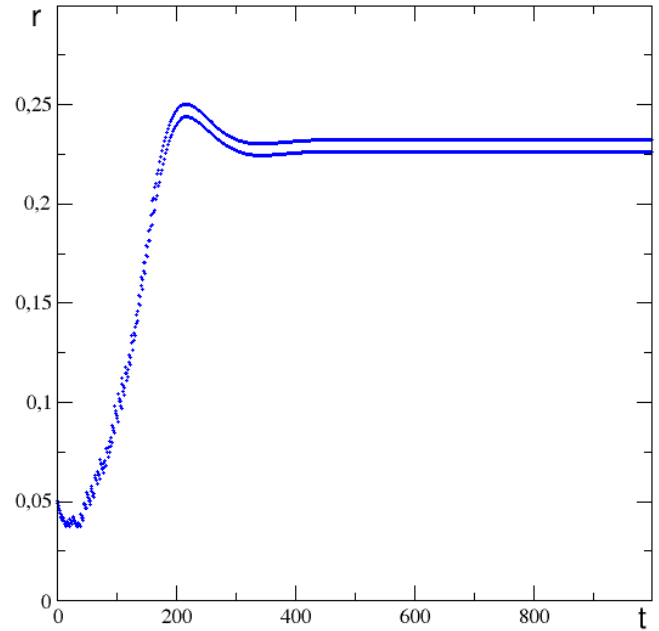

(b)

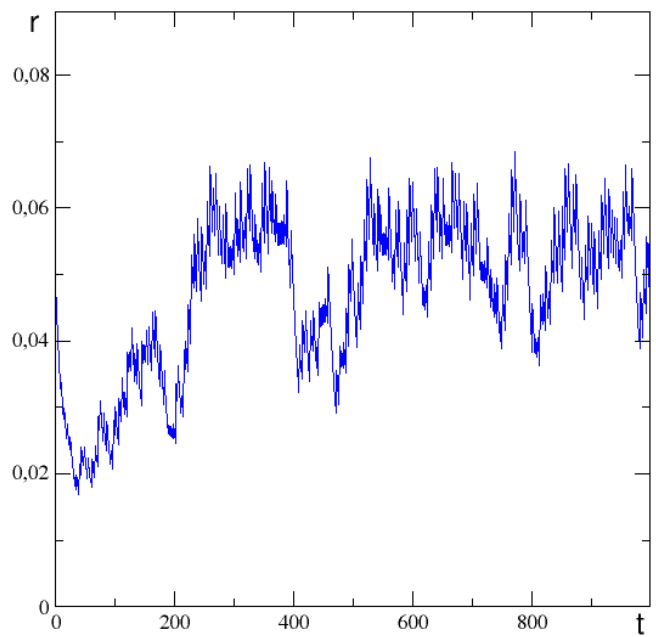

(d)

Figure 16: On the left column two projections on the $x-y$ plane of the trajectory $(x(t), y(t), r(t))$ of the evolutionary oligopoly model. On the right column the dynamics of variable $r$. Upper line $K_{G}=0.025$ and $K_{L}=0$. Bottom line $K_{G}=0.05$ and $K_{L}=0$. Parameters: $N=20, \beta=1, K_{G}=0.05, K_{L}=0, \alpha=0.3, \lambda=1$ and $c=0.1$.

We would like to thank Gian Italo Bischi, Laura Gardini and Fabio Tramontana for helpful discussions on the paper and the participants to the $8^{\text {th }}$ MDEF Workshop in Urbino, September 2014. We are indebted to two anonymous Referees for their valuable advices and very constructive, precise and helpful suggestions. The usual disclaimer applies. This work has been performed under the auspices of COST Action IS1104 "The EU in the new complex geography of economic systems: models, tools and policy evaluation".

\section{References}

[1] E. Droste, C. H. Hommes, and J. Tuinstra. Endogenous fluctuations under evolutionary pressure in Cournot competition. Games and Economic Behavior, 40(2):232-269, 2002. 
[2] C. H. Hommes, M. I. Ochea, and J. Tuinstra. On the stability of the Cournot equilibrium: An evolutionary approach. Working paper, 2011.

[3] R. D. Theocharis. On the stability of the Cournot solution on the oligopoly problem. Review of Economic Studies, 27(2):133-134, 1960.

[4] G. I. Bischi, A. K. Naimzada, and L. Sbragia. Oligopoly games with local monopolistic approximation. Journal of Economic Behavior \&3 Organization, 62(3):371-388, 2007.

[5] J. Tuinstra. A price adjustment process in a model of monopolistic competition. International Game Theory Review, 6(3):417-442, 2004.

[6] G. I. Bischi and A. Naimzada. Advances in Dynamic Games and Applications, chapter Global Analysis of a Duopoly Game with Bounded Rationality, pages 361-385. Birkhauser, 2000.

[7] G. I. Bischi, F. Lamantia, and D. Radi. An evolutionary Cournot model with limited market knowledge. Journal of Economic Behavior \& Organization, 116:219-238, 2015.

[8] A. Kirman. Individual Forecasting and Collective Outcomes, chapter Mistaken beliefs and resultant equilibria. Cambridge University Press, 1983.

[9] V. Brousseau and A. Kirman. Game Theory and Economic Applications, chapter Apparent convergence of learning processes in mis-specified games. Springer, 1992.

[10] M. Anufriev, D. Kopnyi, and J. Tuinstra. Learning cycles in bertrand competition with differentiated commodities and competing learning rules. Journal of Economic Dynamics 83 Control, 37(12):25622581, 2013.

[11] F. Cavalli, A. Naimzada, and F. Tramontana. Nonlinear dynamics and global analysis of a heterogeneous Cournot duopoly with a local monopolistic approach versus a gradient rule with endogenous reactivity. Communications in Nonlinear Science and Numerical Simulation, 23(1-3):245-262, 2015.

[12] J. B. Rosen. Existence and uniqueness of equilibrium points for concave N-person games. Econometrica: Journal of the Econometric Society, 33(3):520-534, 1965.

[13] A. Cabrales and J. Sobel. On the limit points of discrete selection dynamics. Journal of Economic Theory, 57(2):407-419, 1992.

[14] J. Hofbauer and K. Sigmund. Evolutionary game dynamics. Bulletin (New Series) of the American Mathematical Society, 40(4):479-519, 2003.

[15] J. Hofbauer and J. W. Weibull. Evolutionary selection against dominated strategies. Journal of Economic Theory, 71(2):558-573, 1996.

[16] M. Kopel, F. Lamantia, and F. Szidarovszky. Evolutionary competition in a mixed market with socially concerned firms. Journal of Economic Dynamics \&3 Control, 48:394-409, 2014.

[17] T. Puu. Chaos in duopoly pricing. Chaos, Solitons \& Fractals, 1(6):573-581, 1991.

[18] G. I. Bischi, C. Chiarella, M. Kopel, and F. Szidarovszky. Nonlinear Oligopolies: Stability and Bifurcations. Springer-Verlag, 2010.

[19] F. Tramontana, L. Gardini, and T. Puu. Global bifurcations in a piecewise-smooth Cournot duopoly game. Chaos, Solitons $\mathcal{E}$ Fractals, 43(1-12):15-24, 2010.

[20] A. A. Agliari, L. Gardini, and T. Puu. Global bifurcations in duopoly when the Cournot point is destabilized via a subcritical Neimark bifurcation. International Game Theory Review, 8(1):1-20, 2006.

[21] L. Lambertini. Oligopoly with hyperbolic demand: A differential game approach. Journal of Optimization Theory and Applications, 145(1):108-119, 2010. 
[22] F. Lamantia. A nonlinear duopoly with efficient production-capacity levels. Computational Economics, 38(3):295-309, 2011.

[23] M. Plank. Some qualitative differences between the replicator dynamics of two player and $n$ player games. Nonlinear Analysis: Theory, Methods 83 Applications, 30(3):1411-1417, 1997.

[24] N. J. Vriend. An illustration of the essential di!erence between individual and social learning, and its consequences for computational analyses. Journal of economic dynamics $\&$ control, 24(1):1-19, 2000.

[25] G.-I. Bischi, M. Kopel, and A. Naimzada. On a rent-seeking game described by a non-invertible iterated map with denominator. Nonlinear Analysis: Theory, Methods 83 Applications, 47(8):5309-5324, 2001.

[26] G. Gandolfo. Economic Dynamics. Springer, 2010.

[27] S. N. Elaydi. An introduction to difference equations. New York, Springer, 1995.

[28] A. Medio and M. Lines. Nonlinear dynamics. Cambridge University Press, 2001.

[29] R. Dieci, G.I. Bischi, and L Gardini. Routes to complexity in a business-cycle model described by a noninvertible triangular map. Cubo A Mathematical Journal, 5(3):367-396, 2003.

[30] R. Dieci, G. I. Bischi, and L. Gardini. From bi-stability to chaotic oscillations in a macroeconomic model. Chaos, Solitons \& Fractals, 12(5):805-822, 2001.

[31] S. F. Kolyada. On dynamics of triangular maps of the square. Ergodic Theory and Dynamical Systems, 12(4):749-768, 1992.

[32] G.-I. Bischi and F. Lamantia. Nonlinear Dynamical Systems in Economics - CISM Lecture Notes, chapter Coexisting attractors and complex basins in discrete-time economic models, pages 187-231. Springer, 2005.

[33] G.-I. Bischi, L. Gardini, and C. Mira. Maps with a vanishing denominator. A survey of some results. Nonlinear Analysis, 47:2171-2185, 2001.

[34] J. Milnor. On the concept of attractor: Correction and remarks. Communications in Mathematical Physics, 102(3):517-519, 1985.

[35] C. Hommes, T. Kiseleva, Y. Kuznetsov, and M. Verbic. Is more memory in evoluionary selection (de)stabilizing? Macroeconomic Dynamics, 16:335-357, 2012. 